Aus der Klinik für Neurologie

(Direktor Prof. Dr. med. Günther Deuschl)

im Universitätsklinikum Schleswig-Holstein, Campus Kiel

an der Christian-Albrechts-Universität

\title{
KOORDINATIVE GANGSTÖRUNG BEI MIGRÄNE-PATIENTEN
}

\author{
Inauguraldissertation \\ zur \\ Erlangung der Doktorwürde \\ der Medizinischen Fakultät \\ der Christian-Albrechts-Universität zu Kiel
}

vorgelegt von

Lars Timm

aus Aurich

Kiel 2011 
Referent: Prof. Dr. med. Stolze

Korreferent: Prof. Dr. med. Stark

Tag der mündlichen Prüfung: 05.12.2011

zum Druck genehmigt: 05.12.2011 
Meinen Eltern und Geschwistern 


\section{Inhaltsverzeichnis}

$\begin{array}{ll}\text { I. Tabellenverzeichnis } & \text { S. } 1\end{array}$

$\begin{array}{ll}\text { II. Abbildungsverzeichnis } & \text { S.2 }\end{array}$

$\begin{array}{ll}\text { 1. Einleitung } & \text { S. } 3\end{array}$

$\begin{array}{ll}\text { 2. Methodik } & \text { S. } 4\end{array}$

2.1 Untersuchte Personen $\quad$ S. 4

2.1.1 Patienten mit Migräne mit Aura (MA) S. 5

2.1.2 Patienten mit Migräne ohne Aura (MO) S. 5

2.1.3 Kontrollgruppe $(\mathrm{N}) \quad$ S. 5

2.1.4 Patienten mit cerebellären Erkrankungen (CD) S. 5

2.1.5 Ein - und Ausschlusskriterien $\quad$ S. 6

2.2 Klinische Untersuchung $\quad$ S. 8

2.2.1 ICARS-Skala S. 8

2.2.2 Bestimmung der individuellen Gehgeschwindigkeit S. 9

2.3 Ganganalyse S. 10

2.3.1 Versuchsaufbau S. 10

2.3.2 Durchführung S. 12

2.3.3 Datenauswertung S. 13

2.4 Statistische Auswertung S. 14

2.4.1 Graphische Darstellung und Software S. 15

2.4.2 Statistik und Signifikanz S. 16

$\begin{array}{ll}\text { 2.4.3 Korrelationen } & \text { S. } 16\end{array}$

$\begin{array}{ll}\text { 3. Ergebnisse } & \text { S. } 18\end{array}$

3.1 Biometrische Daten $\quad$ S. 18

3.2 ICARS-Score Analyse S. 18

3.3 Analyse des natürlichen Gangbildes $\quad$ S. 19

3.3.1 Korrelationen zu Gangparametern des natürlichen $\quad$ S. 23 Gangbildes 
3.4 Seiltänzergang-Analyse

S. 24

3.4.1 Korrelationen zu Gangparametern des

S. 31

Seiltänzerganges

4. Diskussion

S. 32

4.1 Cerebelläre Gangstörung bei Migräne-Patienten?

S. 32

4.2 Korrelation von Migräne-Variablen und Gangparametern

S. 34

5. Zusammenfassung

S. 36

6. Literaturverzeichnis

S. 38

7. Danksagung

S. 47

8. Lebenslauf

S. 48 


\section{Tabellenverzeichnis}

Tabelle 1: Daten der cerebellär erkrankten Patienten $\quad$ S. 6

$\begin{array}{ll}\text { Tabelle 2: Biometrische Daten } & \text { S. } 18\end{array}$

$\begin{array}{lll}\text { Tabelle 3: } & \text { Ergebnisse der ICARS-Score Analyse } & \text { S. } 18\end{array}$

Tabelle 4: Darstellung aller Schrittparameter der Gruppen N, MO, MA S. 19 im natürlichen Gangbild

Tabelle 5: Darstellung der Schrittparameter im natürlichen Gang (N,M,CD) S. 22

Tabelle 6: Korrelationen von klinischen Daten und Gangparametern $\quad$ S. 24 im natürlichen Gang

Tabelle 7: $\quad$ Darstellung aller Schrittparameter der Gruppen N, MO, MA $\quad$ S. 24 im Seiltänzergang

Tabelle 8: Signifikant unterschiedliche Schrittparameter im Seiltänzergang S. 28 (N,M,CD)

Tabelle 9: Korrelationen von klinischen Daten und Gangparametern im Seiltänzergang 


\section{Abbildungsverzeichnis}

Abbildung 1: Infrarotkamera (240 Hz ProReflex ${ }^{\circledR}$, Qualysis)

S. 10

Abbildung 2: Darstellung der Markerpositionen

S. 11

Abbildung 3: Sequenz einer Probandin beim Seiltänzergang

S. 12

Abbildung 4: Graphische Darstellung signifikant unterschiedlicher

S. 23

Parameter im natürlichen Gang

Abbildung 5: Graphische Darstellung signifikant unterschiedlicher

S. 26

Parameter der Seiltänzergang-Analyse

Abbildung 6: Graphische Darstellung signifikant unterschiedlicher

Parameter der Seiltänzergang-Analyse, 2. Teil

Abbildung 7: Graphische Darstellung signifikant unterschiedlicher

S. 28

Parameter der Seiltänzergang-Analyse (N,M,CD)

Abbildung 8: Graphische Darstellung signifikant unterschiedlicher

S. 29

Parameter der Seiltänzergang-Analyse (N,M,CD) 2.Teil

Abbildung 9: Graphische Darstellung der Zehenmarker als Pendelkurve

S. 30 in der Seiltänzergang-Analyse 


\section{Einleitung}

Obwohl die Migräne gemäß der Klassifikation der International Headache Society die häufigste primäre Kopfschmerzerkrankung ist, gibt es bis heute lediglich Hypothesen und Theorien zur genauen Pathophysiologie und Ätiologie dieses Krankheitsbildes. Relative Einigkeit herrscht in der Fachliteratur darüber, dass genetische Komponenten maßgeblich an der Entstehung und Ausprägung dieser Kopfschmerzerkrankung beteiligt sind.

So beschrieb Russell 1996 anhand von durchgeführten Familienstudien das erhöhte Risiko für Verwandte ersten Grades, ebenfalls von Migräne betroffen zu sein. Bei Migräne mit Aura war das Risiko dabei sogar um das Vierfache erhöht im Vergleich zur Allgemeinbevölkerung. Weitere Hinweise kamen aus der Zwillingsforschung. Die Ergebnisse zeigten jedoch, dass kein einfacher Mendel'scher Erbgang mit vollständiger Penetranz zugrundegelegt werden kann, da die Konkordanz bei eineiigen Zwillingen sonst $100 \%$ sein müsste (Haan et al. 1997).

In einer Publikation vom Mai 2001 beschreibt der Neurologe Sandor aus Zürich bei Migräne-Patienten mit und ohne Aura $(n=35$; Migräne ohne Aura $=16$; Migräne mit Aura $=19$ ) diskrete Auffälligkeiten in einem Zeigeversuch-Paradigma, die sich insbesondere durch eine Dysmetrie als cerebelläre Störung interpretieren lassen. Als Ursache wird eine Mutation cerebellärer $\mathrm{Ca}^{2+}$ Rezeptoren im Kleinhirn angenommen, die auch im ZNS von Patienten mit familiärer hemiplegischer Migräne (FHM) vorkommen. Genaueres müsste laut Sandor durch eine Genanalyse näher verifiziert werden. Ähnliche Ergebnisse koordinativer Auffälligkeiten bei Migräne-Patienten werden in einer Publikation von Harno im Dezember 2003 beschrieben, in der okulomotorische und vestibuläre Untersuchungen sowie neurologische Testungen zur posturalen Instabilität im attackenfreien Intervall durchgeführt wurden.

Basierend auf der Hypothese der cerebellären Dysfunktion bei Migräne wurden wie folgt beschrieben Migräne-Patienten mit Aura (MA; $n=17$ ), ohne Aura (MO; $n=12$ ) sowie eine Kontrollgruppe gesunder Probanden ( $N ; n=19)$ klinisch untersucht. Die Einteilung in die jeweilige Migräne-Gruppe erfolgte nach genauer Anamnese der Migräneform gemäß der Klassifikation der International Headache Society (IHS) von 2004. 
Ziel der Untersuchungen war zu analysieren, ob sich in einer standardisierten Analyse koordinativ komplexer Bewegungsabläufe signifikante Defizite bei Patienten mit Migräne im Vergleich zu gesunden Normalprobanden feststellen lassen. Zu diesem Zweck wurde neben einer klinisch-neurologischen Untersuchung, basierend auf der von Trouillas et al. 1997 entwickelten Skala, eine Ganganalyse mittels Laufband, Infrarot-Kamerasystem und speziell konzipierter Software durchgeführt, deren genauer Aufbau in Kapitel 2.3 erläutert wird. Ergänzend wurden zu weiteren Vergleichen Daten einer Gruppe von Patienten mit cerebellären Erkrankungen (CD; $\mathrm{n}=8$ ) betrachtet, die mit dem gleichen Versuchsaufbau untersucht wurden.

\section{Methodik}

Die Untersuchung der Patienten und Probanden erfolgte prospektiv nach einem standardisierten Verfahren, welches sowohl koordinative und kinematische Funktionen gemäß der Trouillas-Ataxie-Skala als auch eine komplette Ganganalyse (ProReflex® System $240 \mathrm{~Hz}$ ) mittels Infrarotkameras auf einem Laufband beinhaltete. Ort der Studie war das Motoriklabor der Klinik für Neurologie des Universitätsklinikums Schleswig-Holstein (UKSH) Kiel von 2007 bis 2008.

\subsection{Untersuchte Personen}

Die 48 untersuchten Probanden und Patienten wurden in drei Gruppen eingeteilt, bestehend aus gesunden Normalprobanden ( $N$ ), Migränepatienten mit Aura (MA) und ohne Aura (MO). Retrospektiv wurde zu weiteren Vergleichen Daten einer Gruppe von Patienten mit cerebellären Erkrankungen (CD) ausgewertet, die in einer vorhergehenden Studie mit der gleichen Methodik gewonnen wurden (biometrische Daten siehe Kapitel 3.1, Tabelle 1). Alle untersuchten Personen wurden hinreichend über die Studie aufgeklärt und gaben inr Einverständnis.

Die zur Erhebung der Daten notwendige Ganganalyse wurde durch die Ethikkommission des UKSH Kiel genehmigt. 


\subsubsection{Patienten mit Migräne und Aura (MA)}

Die 17 untersuchten Migränepatienten mit Aura (12 Frauen, 5 Männer) waren durchschnittlich 36,4 \pm 13 ,8 Jahre alt. Rekrutiert wurden sie meist telefonisch anhand von Patientenlisten der Klinik für Neurologie des Universitätsklinikums SchleswigHolstein zu Kiel, so fern sie dort in den vergangenen Jahren untersucht und behandelt wurden. Einige weitere Personen aus dem Kollegen- und Freundeskreis erklärten sich ebenfalls zur Teilnahme bereit.

\subsubsection{Patienten mit Migräne ohne Aura (MO)}

Die 12 untersuchten Personen mit einer Migräne ohne Aura (9 Frauen, 3 Männer) waren durchschnittlich im Alter von 32,9 \pm 8,0 Jahren. Ihre Rekrutierung erfolgte auf dieselbe Weise wie bereits bei den Migränepatienten mit Aura beschrieben.

\subsubsection{Kontrollgruppe $(N)$}

Als Vergleichsgruppe wurden 19 gehgesunde Erwachsene aus dem Kollegen- und Freundeskreis rekrutiert, die ein Durchschnittsalter von 35,4 \pm 13,1 Jahren hatten (12 Frauen, 7 Männer).

\subsubsection{Patienten mit cerebellären Erkrankungen (CD)}

Die Daten der 8 Personen mit cerebellären Erkrankungen, welche retrospektiv zum Vergleich der koordinativen Auffälligkeiten herangezogen wurden, hatten ein Durchschnittsalter von 52,5 \pm 17,3 Jahren (1 Frau, 7 Männer). Wichtig für die Korrektheit des Vergleichs ist dabei die Tatsache, dass die im Jahr 2000 erhobenen Daten unter exakt den gleichen Bedingungen bestimmt wurden wie die der Migräneund Kontrollgruppen in dieser klinischen Studie. Die unterschiedlichen Ätiologien der Erkrankungen sind der folgenden Tabelle zu entnehmen. 


\begin{tabular}{|c|c|c|c|}
\hline Alter in Jahren & Geschlecht & Erkrankungsdauer in Jahren & $\begin{array}{c}\text { Ätiologie } \\
\text { OPCA }\end{array}$ \\
\hline 69 & Mann & 1 & $\begin{array}{c}\text { Lindau Tumor mit } \\
\text { cerebellärer Blutung }\end{array}$ \\
\hline 84 & Frau & 2 & $\begin{array}{c}\text { Alkohol toxische } \\
\text { Degeneration }\end{array}$ \\
\hline 46 & Mann & 1 & OPCA \\
\hline 37 & Mann & 1 & SCA Typ 2 \\
\hline 49 & Mann & 6 & SCA Typ 3 \\
\hline 39 & Mann & 14 & EOCA \\
\hline 36 & Mann & 13 & -- \\
\hline 59 & Mann & & Cerebelläre Metastase \\
\hline
\end{tabular}

Tabelle 1: Daten der cerebellär erkrankten Patienten OPCA = Multisystematrophie vom olivopontocerebellären Typ; SCA = Spinocerebelläre Ataxie; EOCA = "early onset cerebellar ataxia"

\subsubsection{Ein - und Ausschlusskriterien}

Zum Einschluss der Personen mit Migräne war es zwingend notwendig, dass ihre Kopfschmerzattacken eindeutig gemäß der IHS klassifiziert werden konnten, um sie anschließend der entsprechenden Untergruppe zuordnen zu können (MA oder MO). Des Weiteren wurden alle Personen von der Studie ausgeschlossen, egal ob gesund oder an Migräne erkrankt, die in irgend einer Form andere neurologische oder orthopädische Erkrankungen aufwiesen oder unter Medikamenteneinfluss standen, welche eine Einschränkung in den sensorischen Sinnesleistungen verursachen könnten. Teilnehmer wurden ebenfalls ausgeschlossen, wenn sie einen oder mehrere der aufgeführten Befunde aufwiesen:

$>$ Manifeste Hemiparese;

> Zentral oder peripher bedingte Tetraparese;

> Zentral oder peripher bedingte Monoparese eines Beines;

> Jegliche Teilparese im Bereich einer unteren Extremität infolge einer segmentalen oder peripheren Nervenläsion;

> Systemdegeneration des zentralen oder peripheren Nervensystems; 
> Idiopathisches oder symptomatisches (insbesondere medikamentös induziertes) Parkinson Syndrom;

$>$ Epilepsie;

> Befund einer unilateralen Kleinhirnläsion oder Anamnese oder Befund einer bilateralen Kleinhirnaffektion;

$>$ Anamnese oder Befund einer entzündlichen oder tumorösen Erkrankung des Gehirns, des Rückenmarks oder ihrer Häute;

> Anamnese einer Hirnkontusion oder offenen Schädelhirnverletzung;

$>$ Befund eines akuten Hirninfarktes;

> Anamnese oder Befund einer intrazerebralen Blutung jeglicher Ätiologie;

> Jegliche Form von Aphasie;

> Myelopathie jeglicher Ätiologie;

> Myopathie jeglicher Ätiologie;

$>$ Sehschwäche über $5 / 50$;

$>$ Hemianopsie;

> Hörstörung, die das Verständnis der Unterhaltungssprache über eine Entfernung von ca. einem Meter nicht mehr erlaubt;

> Anamnese oder Vorliegen einer Alkohol- oder Medikamentenabhängigkeit;

> Einnahme von Neuroleptika, Antidepressiva oder Benzodiazepinen während des Tages;

> M. Bechterew, Torsionsskoliose oder andere Deformationserkrankungen der Wirbelsäule;

$>$ Lumbago oder Lumboischialgie;

> Schmerzzustände in Hüft- und/oder Knie- und/oder Sprunggelenk, die sich beim Geradeaus- oder Treppenabgehen manifestieren;

> Schmerzzustände in den Beinen oder Füßen beim Stehen, die kontinuierliches Gehen einer Strecke von mehr als $100 \mathrm{~m}$ regelmäßig verhindern;

$>$ Anamnese einer Fraktur des Hüft-, Knie- oder Sprunggelenks, Femurs Fußgewölbes, der Tibia oder Fibula im Verlauf der letzten 12 Monate vor der Untersuchung;

Zustand nach Implantation einer Gelenkprothese im Bereich einer oder beider Hüft-, Knie- oder Sprunggelenke oder Versteifung in einem oder mehrerer dieser Gelenke; 
> Jegliche Amputation oder Teilamputation an Armen oder Beinen;

> Manifestes Ulcus cruris;

$>$ Elephantiasis;

> Hammerzehen, Clavi oder Fußdeformitäten, die als Ursache von Schmerzen oder unphysiologischem Bewegungsablauf mit dem Gangbild interferieren;

> Beinlängendifferenz von mehr als $1,5 \mathrm{~cm}$;

$>$ Diabetes mellitus, der nicht diätetisch beherrschbar ist;

> Asthma bronchiale;

> Globalinsuffizienz des Herzens (gemeinsames Vorliegen von Knöchelödemen und Ruhedyspnoe);

> Implantierter Herzschrittmacher;

\subsection{Klinische Untersuchung}

Die Untersuchung der Probanden und Patienten erfolgte für alle Gruppen (MA, MO, N) nach dem gleichen, standardisierten Schema. Vorausgehend für die Migränepatienten war lediglich eine genaue Anamnese gemäß der IHSKlassifikation, um die Gruppenzugehörigkeit zu bestimmen. Außerdem wurde darauf geachtet, dass sich die Migräne-Patienten im attackenfreien Intervall befanden und keinerlei Dauer- oder prophylaktische Medikation zu sich nahmen.

\subsubsection{ICARS-Skala}

Die International Cooperative Ataxia Rating Scale (ICARS) ist das Ergebnis von zahlreichen multizentrischen und internationalen Untersuchungen über einen langen Zeitraum. Sie ermöglicht eine quantitative Bewertung und Objektivierung von Haltungsfehlern, Ataxien, Dysarthrien sowie okulomotorischen Störungen mittels eines präzise definierten Punkte-Scores (Trouillas et al. 1997). Die dabei maximal erreichbaren 100/100 Punkte wären das Ergebnis mit dem höchst möglichen Beeinträchtigungsgrad, ein Score von 0/100 hingegen wäre das Ergebnis einer völlig unauffälligen Untersuchung. Der Teilbereich „Haltung und Gang“ berücksichtigt die Qualitäten Gehfähigkeit (10 Meter Teststrecke inklusive Drehung), Gehgeschwindigkeit, Stehfähigkeit, Fußabstand im sicheren (selbst gewählten) Stand, Körperschwankungen bei geschlossenen Füßen und 
geöffneten/geschlossenen Augen sowie in der Sitzposition (Schenkel zusammen, harter Sitz, Arme gekreuzt). Die Überprüfung der kinetischen Funktionen erfolgte durch den Knie-Hacke-Test, Finger-Nase-Test (Störung des Bewegungsablaufs, Dysmetrie oder Intentionstremor), Finger-Finger-Test sowie der Beurteilung von Diadochokinese und dem Spiralenzeichnen. Sprechstörungen wurden im Anamnesegespräch und durch das Wiederholen eines Standardsatzes auf die Flüssigkeit und Klarheit der Sprache hin bewertet. Die Qualität der Okulomotorik wurde anhand von Blickfolgebewegungen, Sakkaden und dem Vorliegen eines Nystagmus beurteilt. Insbesondere wurden die Patienten auf das Vorliegen einer Sakkaden-Dysmetrie untersucht. Das Zusammentragen der Ergebnisse dieser Teilbereiche ergibt dann den beschriebenen Punkte-Score, der eine Standardisierung koordinativer Defizite ermöglicht.

\subsubsection{Bestimmung der individuellen Gehgeschwindigkeit}

Um die Ganganalyse mittels ProReflex® System $(240 \mathrm{~Hz})$ durchführen zu können, war es zunächst notwendig, die individuelle Gehgeschwindigkeit der Probanden im natürlichen Gang sowie im Seiltänzergang über Grund zu ermitteln.

Zu diesem Zweck hatten alle untersuchten Personen eine definierte Gehstrecke von fünf Metern zurückzulegen. Dabei wurde mittels Lichtschranken die Zeit gestoppt. Das Lichtschrankenpaar wurde dafür standardisiert in Höhe der distalen Oberschenkel positioniert, um ein zu frühes Auslösen der Zeitmessung durch Pendelbewegungen der Arme zu verhindern. Diese Messung wurde jeweils im natürlichen Gang und im Seiltänzergang dreimal wiederholt, so dass nach Bestimmung des jeweiligen Mittelwerts die durchschnittliche Zeit für beide Modalitäten feststand. Mittels der Formel $1 / x$ mal 18 wurden diese Zeiten anschließend in Gehgeschwindigkeiten, also in Kilometer pro Stunde, umgerechnet. Diese individuell bestimmten Tempi wurden bei der anschließenden Ganganalyse auf dem Laufband eingestellt, so dass jeder Proband in dem eigenen und für inn typischen Tempo laufen konnte. Die Berücksichtigung der individuellen Gehgeschwindigkeit hat damit in den folgenden Versuchen geholfen, Fehltritte oder andere Abweichungen aufgrund ungewohnter Gehgeschwindigkeiten zu vermeiden. 


\subsection{Ganganalyse}

\subsubsection{Versuchsaufbau}

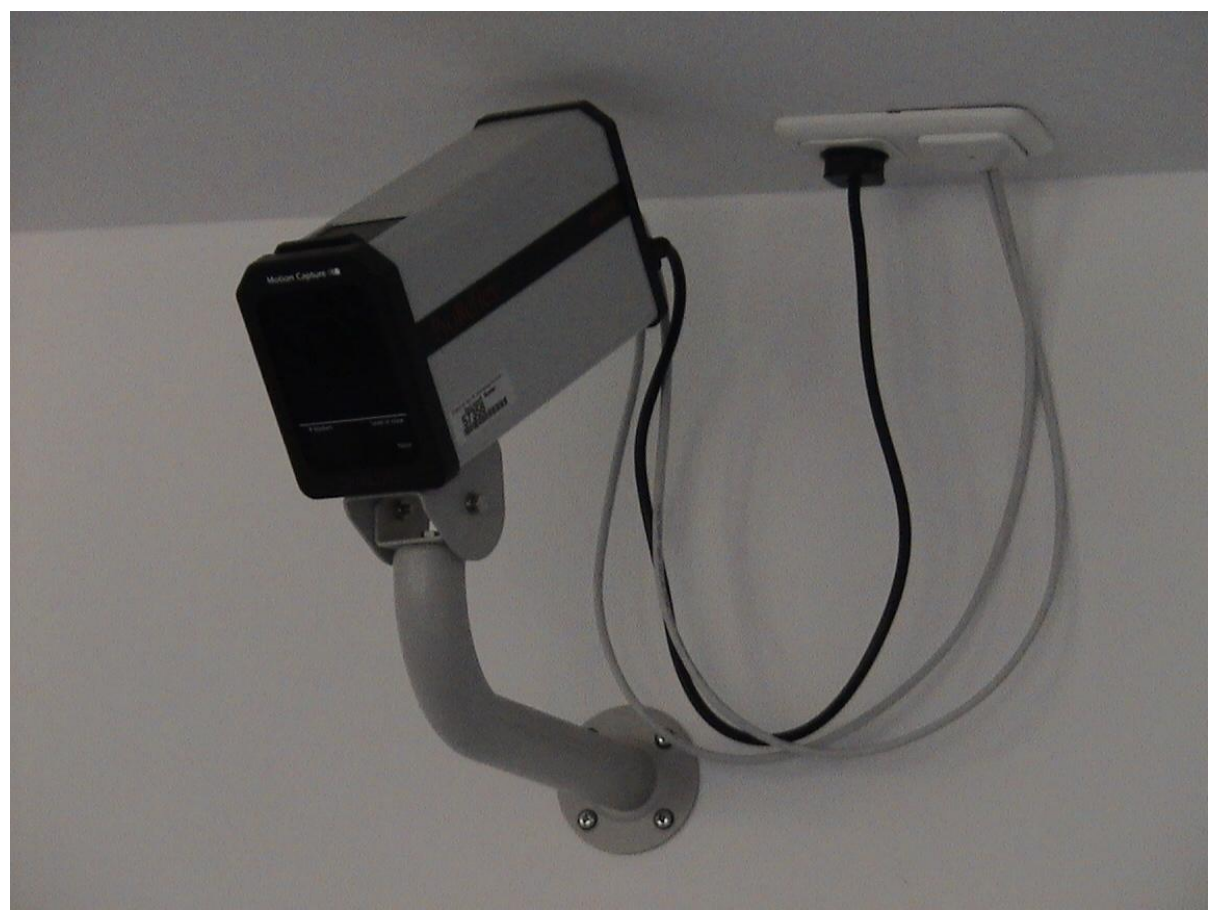

Abb. 1: Infrarotkamera (240 Hz ProReflex $\AA$, Qualysis)

Um das Gangbild der Probanden kinematisch untersuchen zu können, wurden im Motoriklabor Infrarotkamerasysteme (240 Hz ProReflex®) der Firma Qualysis aus Schweden installiert, die bei einer entsprechenden Ausstattung der zu untersuchenden Person mit reflektierenden Markern in der Lage sind, Bewegungen der Marker im dreidimensionalen Raum zu messen.

Die dafür verwendeten sechs Kameras wurden in den vier Ecken des Raumes sowie jeweils eine rechts und links an den Seitenwänden des Labors montiert, deren gemeinsamer Fokus die Raummitte und das dort befindliche Laufband darstellte. Eine Verkopplung der Infrarotkameras untereinander sowie die Verbindung mit einem Computer mit der entsprechenden Software ermöglichen dabei den Datenzusammenschluss der einzelnen Systeme. 
Durch diese Positionierung der Kameras und nach standardisierter Kalibrierung des Systems war es möglich, ein Objekt oder eine Person, die mit reflektierenden Markern versehen wurde, bei der Bewegung im Raum zu filmen und später zu analysieren. Um reliable Ergebnisse der Bewegungsanalyse der Beine während des Gehens zu gewährleisten, wurden vierzehn anatomische Messpunkte bestimmt, die bei jeder zu untersuchenden Person mit einem reflektierenden Marker versehen wurden. Die Marker bestanden aus einem runden Kunststoffträger (18 Milimeter Durchmesser), der mit einer Infrarotlicht reflektierenden Folie überzogen war. Die anatomischen Punkte waren jeweils rechts und links die Spina iliaca anterior superior, der Trochanter major, der distale Oberschenkel, der laterale Kniegelenkspalt, der Malleolus lateralis, der laterale Calcaneus sowie das Os metatarsale des Digitus minimus. Eine Analyse dieser Punkte in Relation zueinander ermöglicht dem Untersucher somit die Bestimmung des Hüft-, Knie- und Sprunggelenkwinkels nach dem Aufnehmen einer Gangsequenz.

14-Marker-Setup
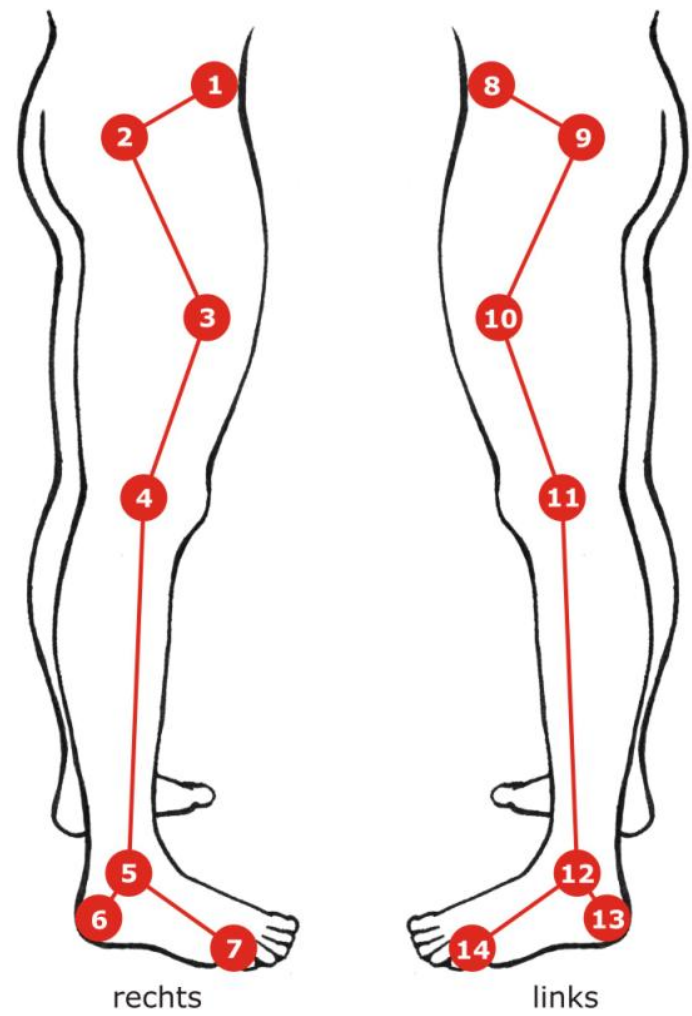

Abb. 2: Darstellung der Markerpositionen 


\subsubsection{Durchführung}

Um die Messpunkte möglichst nahe am Körper positionieren zu können, wurden für die Versuchspersonen von der Klinik hautenge Sporthosen gestellt. Die Füße waren durch enge Sportsocken bekleidet. Nach dem Aufbringen der 14 Marker bekamen die Probanden einen Zeitraum von fünf Minuten, um sich an das Gehen auf dem Laufband zu gewöhnen. Das Tempo entsprach dabei wie bereits beschrieben der individuell bestimmten Gehgeschwindigkeit. Alle teilnehmenden Personen wurden dazu angehalten, die Arme beim Gehen vor dem Bauch zu verschränken, da ansonsten die Pendelbewegungen der Arme die Erfassung der oberen Hüftmarker durch die Kameras erschwert und verfälscht hätte. Es wurden pro Proband (je nach Aufzeichnungsqualität) mehrere Gangsequenzen von 20 Sekunden Dauer aufgezeichnet, jeweils im natürlichen Gang sowie im Seiltänzergang. Parallel wurden die Messdurchgänge mit einer an den Steuerungs-Computer gekoppelten digitalen Videokamera aufgezeichnet. Zur Ermittlung der Gangparameter im Seiltänzergang wurde auf der Mitte des Laufbandes eine Linie mit $2 \mathrm{~cm}$ breitem, hellrotem Isolierklebeband markiert, auf der die Probanden laufen sollten. Zusätzlich wurden während des Seiltänzerganges die Fehltritte (missteps) pro Minute über eine Zeit von insgesamt drei Minuten registriert. War beim Auftreten die Fläche des gesamten Fußes neben der $2 \mathrm{~cm}$ breiten Markierungslinie, wurde dies als Fehltritt gewertet.

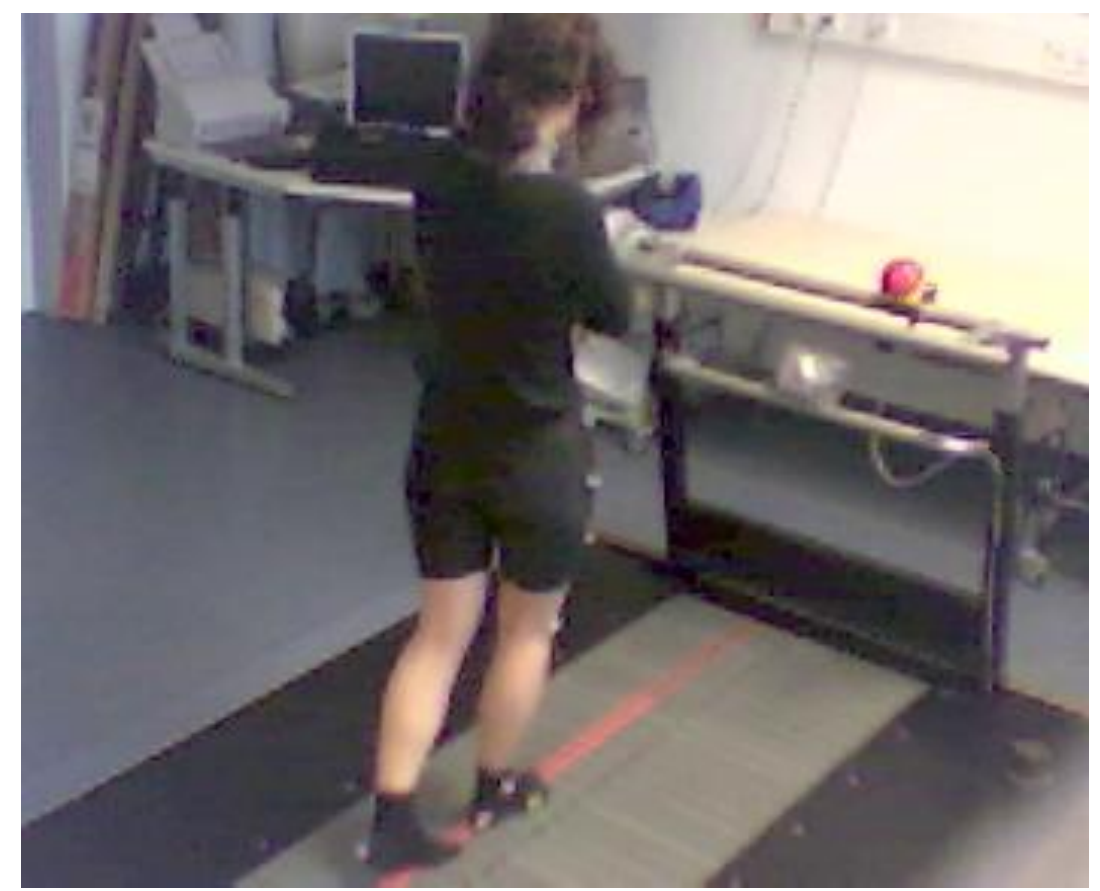

Abb. 3: Sequenz einer Probandin beim Seiltänzergang (Ausschnitt Digitalkamera) 
Den Versuchspersonen war es jederzeit möglich, das Laufband durch drücken eines am vorderen Geländer montierten Knopfes selbstständig zu stoppen oder durch den Versuchsleiter stoppen zu lassen. Stark gehbehinderte Patienten, die als sturzgefährdet eingeschätzt wurden, konnten sich am vorderen Geländer festhalten und wurden zusätzlich durch ein an der Decke des Raumes befestigtes Gurtungssystem gesichert, das ein freies Laufen ermöglichte und gleichzeitig dem Patienten die nötige Sicherheit bot, im Falle eines Stolperns einen Sturz zu verhindern. Der Sicherheitsgurt war dabei ohne Gewichtsentlastung eingestellt.

\subsubsection{Datenauswertung}

Zur weiteren Bearbeitung mussten die mittels Qualysis $\circledast$ Software ermittelten Daten der 20 sekündigen Infrarotkamera-Aufzeichnungen in ein für die vom Institut eigens entwickelte Software (Gaitlab) verwertbares Format transferiert werden. Mit Hilfe dieser Daten, die für jeden Marker zu allen Zeitpunkten der 20 Sekunden-Aufnahme $x, y$ und $z$-Koordinaten darstellen, war es nun möglich, detaillierte Berechnungen von Gangparametern durchzuführen. Grundlegend für alle folgenden Berechnungen war die Einteilung der 20 sekündigen Laufsequenzen in einzelne Schritte. Dabei wurde der Aufsetz- und der Abhebezeitpunkt jedes Fußes einzeln bestimmt. Die Bestimmung der Zeitpunkte erfolgt anhand der im Setup und der Kalibrierung des Qualysis-Programms festgelegten Koordinaten sowie durch die Aufzeichnungen des Zehen- und des Fersenmarkers. Dieses Vorgehen hat sich in der Praxis als sehr zuverlässig erwiesen.

Die im Vorversuch erhobene individuelle Gehgeschwindigkeit wird zur Auswertung manuell eingegeben und zusätzlich noch durch das Gaitlab-Programm berechnet. Die vom Programm berechnete Laufbandgeschwindigkeit dient dabei lediglich der Eingabekontrolle und zur Vermeidung von Fehlern. Zur Berechung der Knie-, Sprung- und Hüftgelenkswinkel werden die jeweilig definierten Marker in Relation gesetzt. Der Kniegelenkswinkel ist somit der Winkel zwischen dem Knöchelmarker, dem Kniemarker und dem Oberschenkelmarker. Der Sprunggelenkswinkel ist der Winkel zwischen dem Zehenmarker, dem Knöchelmarker und dem Kniemarker. Der Hüftgelenkswinkel ist der Winkel zwischen dem Marker an der Spina iliaca anterior superior, dem Trochantermarker und dem Oberschenkelmarker. 
Des Weiteren wurden die Schritthöhe und die Schrittbreite bestimmt. Zur Bestimmung der Schrittbreite wurde zunächst die Entfernung beider Füße in der xAchse während aller Doppelstandphasen ermittelt, also in allen Perioden, in denen beide Fersen den Boden berührten. Die Entfernung der Fersenmarker, unter Berücksichtigung der Absatzmittelpunkte, ergab somit die Breite des Schrittes während der Doppelstandphase. Neben der Standphase wurde auch die Schwungphase ermittelt, welche definiert ist als Zeit vom Abheben eines Fußes bis zum Aufsetzen desselben Fußes.

Ein wichtiger Parameter der Analyse ist der Ataxie-Quotient, der einen exakten Vergleich der Abweichungen der gemessenen Fußbewegung von der optimalen Wegstrecke ermöglicht. Dabei wird die Strecke der Zehenmarker eines Fußes vom Abheben bis zum nächsten Aufsetzen desselben bestimmt. Die kürzeste Verbindung dieser beiden Punkte stellt demnach die optimale Wegstrecke dar. Der Quotient aus optimaler Wegstrecke und der davon abweichenden realen Wegstrecke wird als Ataxie-Quotient bezeichnet.

Unabhängig von der Gaitlab-Auswertung war einer der entscheidenden Parameter der Ganganalyse die erhobenen Fehltritte (siehe Kapitel 2.3.2), die bei allen 48 Probanden und Patienten von demselben Untersucher bewertet und protokolliert wurden.

\subsection{Statistische Auswertung}

Die Untersuchung der einzelnen Probanden und Patienten erfolgte wie beschrieben standardisiert und unabhängig der Gruppenzugehörigkeit nach dem gleichen Schema. Alle Versuchspersonen absolvierten die Koordinationstestungen zur Ermittlung des ICARS-Score's, die Bestimmung der individuellen Gehgeschwindigkeit sowie die Ganganalyse mittels Laufband im natürlichen- und im Seiltänzergang.

Zur statistischen Auswertung wurden die 48 Probanden, wie in Kapitel 2.1 beschrieben, in die Gruppen N, MO, MA eingeteilt. Aus mehreren individuellen Messungen wurde jeweils der Durchgang zur Auswertung ausgewählt, der die größte Messgenauigkeit aufwies. Dabei war entscheidend, dass zu mehreren Schrittphasen alle 14 Marker vollständig von den sechs Infrarot-Kameras erfasst wurden, um eine qualitative Beurteilung der Schrittsequenz im Gaitlab-Programm zu ermöglichen. Für 
die Analyse der Variablen der Einzelschritte wurden in allen Fällen die Daten des rechten Beines verwendet.

In die statistische Auswertung eines jeden Teilnehmers wurden folgende Parameter und Variablen der Ganganalyse einbezogen:

Der ICARS-Score;

Mittelwert, Standardabweichung und Variationskoeffizient der Gehgeschwindigkeit $(\mathrm{m} / \mathrm{s})$;

Mittelwert, Standardabweichung und Variationskoeffizient der Doppelschrittlänge (cm);

Mittelwert, Standardabweichung und Variationskoeffizient der Teilschrittlänge $(\mathrm{cm})$;

Der Symmetriequotient (\%);

Mittelwert, Standardabweichung und Variationskoeffizient der Standphasendauer (ms);

Mittelwert, Standardabweichung und Variationskoeffizient der Schwungphasendauer (ms);

Mittelwert, Standardabweichung und Variationskoeffizient der

Doppelstandphasendauer (ms);

Die Kadenz (Anzahl der Schritte pro Minute);

Mittelwert, Standardabweichung und Variationskoeffizient der Schrittbreite $(\mathrm{mm})$;

Mittelwert, Standardabweichung und Variationskoeffizient der Schritthöhe $(\mathrm{cm})$;

Die missteps (Anzahl der Fehltritte pro Minute);

Der Ataxie-Quotient (Quotient aus tatsächlicher und kürzester Weglänge);

Mittelwert, Standardabweichung und Variationskoeffizient des Fußwinkels (Grad);

Mittelwert des maximalen und minimalen Hüftgelenkwinkels (Grad);

Mittelwert des maximalen und minimalen Kniegelenkwinkels (Grad);

Mittelwert des maximalen und minimalen Fußgelenkwinkels (Grad).

\subsubsection{Graphische Darstellung und Software}

Alle statistischen Analysen wurden auf einem Computer mit dem Programmpaket SPSS 10.0 (Statistical Package for the Social Sciences, SPSS Inc.) durchgeführt und berechnet. Zur graphischen Darstellung signifikanter Parameter im Säulendiagramm wurde das Programm Excel von Microsoft verwendet. Die Graphiken zur Darstellung der "Leuchtspuren“ der zyklischen Gangbewegung (kontinuierliche Verfolgung 
Marker 7 und 14) im Seiltänzergang in Abbildung 5 wurden mit Hilfe des Gaitlab 2.2Programms generiert.

\subsubsection{Statistik und Signifikanz}

Mit Hilfe des SPSS-Programmes wurde aus den ermittelten Datensätzen zunächst eine deskriptive Statistik erstellt. Berechnet wurden der Mittelwert sowie die Standardabweichung der einzelnen Parameter. Des Weiteren erfolgte die Bestimmung des Variationskoeffizienten für ausgewählte Parameter. Als Quotient der Division der Standardabweichung geteilt durch den Mittelwert wird der Variationskoeffizient in Prozent angegeben und ist ein $\mathrm{Maß}$ für die Streuung der Einzelergebnisse um den berechneten Mittelwert.

Zur Überprüfung signifikanter Unterschiede in den Ganganalysen der einzelnen Gruppen wurden nicht-parametrische Tests durchgeführt. Dabei wurde ein gemeinsames Signifikanzniveau von $\alpha \leq 0,05$ festgelegt.

Mit Hilfe des parameterfreien Kruskal-Wallis-Test wurden zunächst die von einander unabhängigen Datensätze der untersuchten Gruppen verglichen und hinsichtlich möglicher signifikanter Unterschiede einzelner Variablen geprüft. Somit wurde ermittelt, ob mögliche Abweichungen lediglich zufällig sind oder einer gemeinsamen Grundgesamtheit entstammen.

Nach Ermittlung der signifikanten Parameter wurden diese im Mann-Whitney U-Test erneut analysiert. Dieser Test ist ebenfalls parameterfrei und ermöglicht den Vergleich zweier unabhängiger Datensätze miteinander. Es wurden jeweils zwei Gruppen (beispielsweise Gruppe N mit Gruppe MO) bezüglich eines in der ersten Testung signifikanten Parameters untersucht.

\subsubsection{Korrelationen}

Um beurteilen zu können, ob signifikante Korrelationen zwischen Variablen der Migräne-Erkrankung und der Ausprägung bestimmter, in den vorangegangenen Berechnungen auffälliger, Gangparameter oder Skalenergebnisse bestehen, wurde im folgenden Pearson's r-Test durchgeführt. Dies geschah sowohl mit den Variablen des natürlichen Gangbildes als auch mit denen des Seiltänzerganges. Relevante 
Daten der Ganganalyse waren dabei der Ataxie-Quotient, die Fehltritte sowie die Schrittbreite der Probanden. Diese wurden auf ihren Zusammenhang zur Dauer der Migräneerkrankung, der Attackenfrequenz, der Attackendauer, der subjektiv beurteilten Intensität sowie die während der Migräneattacken auftretenden Begleiterscheinungen auf ihre Signifikanz überprüft. Dieser Test wurde nur mit den Daten der an Migräne erkrankten Probanden der Gruppen MO und MA durchgeführt. Pearson's Korrelationskoeffizient kann Werte zwischen -1 und +1 annehmen, wobei ein Wert von +1 (oder -1) einen linearen Zusammenhang zweier Variablen darstellen würde. Das festgelegte Signifikanzniveau betrug $p \leq 0,05$. 


\section{Ergebnisse}

\subsection{Biometrische Daten}

\begin{tabular}{|c|c|c|c|c|}
\hline & $\mathbf{N}$ & MO & MA & CD \\
\hline Alter (Jahren) & $35,4 \pm 13,1$ & $32,9 \pm 8,0$ & $36,4 \pm 13,8$ & $52,5 \pm 17,3$ * \\
\hline $\begin{array}{c}\text { Körpergröße } \\
(\mathrm{cm})\end{array}$ & $173,9 \pm 9,1$ & $174,9 \pm 9,1$ & $172,3 \pm 8,1$ & $171,6 \pm 6,1$ \\
\hline $\begin{array}{c}\text { Körpergewicht } \\
(\mathrm{kg})\end{array}$ & $67,3 \pm 12,8$ & $69,2 \pm 12,6$ & $75,3 \pm 17,4$ & $73,6 \pm 6,5$ \\
\hline Beinlänge (cm) & $91,3 \pm 11,5$ & $94,2 \pm 7,2$ & $92,6 \pm 4,5$ & $92,5 \pm 4,3$ \\
\hline
\end{tabular}

Tabelle 2: Biometrische Daten. Signifikante Unterschiede $(p \leq 0,05)$ sind gekennzeichnet $\left(^{*}\right)$.

Bei Betrachtung der Altersstruktur der einzelnen Gruppen fällt ein signifikant erhöhtes Durchschnittsalter der retrospektiv untersuchten Patienten mit cerebellären Erkrankungen auf. Beim Vergleich der übrigen Parameter (Körpergröße, Körpergewicht und Beinlänge) ließen sich keine signifikanten Unterschiede zwischen den untersuchten Gruppen feststellen.

\subsection{ICARS-Score Analyse}

\begin{tabular}{|c|c|c|c|}
\hline & $\mathbf{N}$ & MO & MA \\
\hline ICARS-Score & $1,05 \pm 0,9$ & $1,92 \pm 1,9$ & $2,6 \pm 2,4^{\text {** }}$ \\
\hline
\end{tabular}

Tabelle 3: $x / 100$ Punkten. Signifikante Unterschiede $(p \leq 0,01)$ sind gekennzeichnet $\left({ }^{* *}\right)$.

In der klinischen Untersuchung zur Beurteilung koordinativer Defizite gemäß der von Trouillas 1997 entwickelten Ataxie-Skala wurden alle Probanden, wie in Kapitel 2.2.1 beschrieben, anhand eines standardisierten Verfahrens bewertet. In der Testung der einzelnen Gruppen gegeneinander mittels Mann-Whitney-Test ergibt sich ein signifikanter Unterschied $(p=0,010)$ zwischen den Ergebnissen der Normalprobanden 
und den Migräne-Patienten (MO+MA). Durch Vergleiche der einzelnen Gruppen untereinander ergibt sich ein noch höheres Signifikanzniveau von $p=0,005$ zwischen den Gruppen N und MA (siehe Tabelle 3). Alle weiteren Vergleiche (N/MO $p=0,154$; MO/MA $p=0,347$ ) ergeben keine signifikanten Unterschiede.

Zusammenfassend erzielen demnach die untersuchten Migräne-Patienten mit Aura (MA) ein relevant schlechteres Resultat in der koordinativen Testung gemäß dem Trouillas-Ataxie-Score. Migräne Patienten ohne Aura sind zwar im Mittel schlechter als die gesunden Vergleichsprobanden $(\mathrm{N})$, jedoch ist dieser Unterschied nicht signifikant.

\subsection{Analyse des natürlichen Gangbildes}

\begin{tabular}{|c|c|c|c|}
\hline Schrittparameter & $\mathbf{N}$ & MO & MA \\
\hline Gehgeschwindigkeit (m/s) & $1,19 \pm 0,15$ & $1,19 \pm 0,06$ & $1,25 \pm 0,13$ \\
\hline $\begin{array}{c}\text { Variationskoeffizient der } \\
\text { Gehgeschwindigkeit (\%) }\end{array}$ & $4,7 \pm 1,8$ & $4,9 \pm 2,5$ & $4,3 \pm 0,7$ \\
\hline Doppelschrittlänge (cm) & $125,3 \pm 13,3$ & $127,3 \pm 7,3$ & $127,0 \pm 15,4$ \\
\hline $\begin{array}{c}\text { Variationskoeffizient der } \\
\text { Doppelschrittlänge (\%) }\end{array}$ & $2,4 \pm 2,5$ & $2,0 \pm 0,9$ & $2,1 \pm 1,0$ \\
\hline Teilschrittlänge (cm) & $62,8 \pm 6,1$ & $62,8 \pm 4,4$ & $63,1 \pm 8,5$ \\
\hline $\begin{array}{c}\text { Variationskoeffizient der } \\
\text { Teilschrittlänge (\%) }\end{array}$ & $3,4 \pm 1,8$ & $4,6 \pm 2,5$ & $4,9 \pm 6,5$ \\
\hline Symmetriequotient (\%) & $1,0 \pm 0,04$ & $1,0 \pm 0,1$ & $1,0 \pm 0,1$ \\
\hline Standphasendauer (ms) & $648,3 \pm 43,7$ & $641,8 \pm 28,7^{\circ}$ & $611,9 \pm 46,0^{\circ}$ \\
\hline $\begin{array}{c}\text { Variationskoeffizient der } \\
\text { Standphasendauer (\%) }\end{array}$ & $2,3 \pm 0,5$ & $2,4 \pm 0,9$ & $2,8 \pm 1,2$ \\
\hline Schwungphasendauer (ms) & $408,5 \pm 15,3$ & $420,1 \pm 21,0$ & $402,6 \pm 24,8$ \\
\hline $\begin{array}{c}\text { Variationskoeffizient der } \\
\text { Schwungphasendauer (\%) }\end{array}$ & $2,1 \pm 0,6$ & $2,4 \pm 0,7$ & $2,9 \pm 1,4$ \\
\hline Doppelstandphasendauer (ms) & $119,5 \pm 16,3^{* *}$ & $113,0 \pm 11,3^{\circ}$ & $104,4 \pm 12,1^{* * \circ}$ \\
\hline $\begin{array}{c}\text { Variationskoeffizient der } \\
\text { Doppelstandphasendauer (\%) }\end{array}$ & $214,5 \pm 890,4$ & $392,8 \pm 1320,9$ & $943,1 \pm 2007,0$ \\
\hline Kadenz & $113,8 \pm 5,9$ & $111,1 \pm 4,0$ & $115,1 \pm 6,6$ \\
\hline Schrittbreite (mm) & $127,3 \pm 25,1$ & $120,6 \pm 40,5$ & $141,3 \pm 28,6$ \\
\hline
\end{tabular}




\begin{tabular}{|c|c|c|c|}
\hline $\begin{array}{c}\text { Variationskoeffizient der } \\
\text { Schrittbreite (\%) }\end{array}$ & $13,2 \pm 4,0$ & $19,8 \pm 9,4$ & $12,9 \pm 5,2$ \\
\hline Schritthöhe (mm) & $204,4 \pm 18,9$ & $202,9 \pm 14,3$ & $207,3 \pm 21,7$ \\
\hline $\begin{array}{c}\text { Variationskoeffizient der } \\
\text { Schritthöhe (\%) }\end{array}$ & $4,5 \pm 3,3$ & $5,5 \pm 5,5$ & $2,7 \pm 1,6$ \\
\hline Fußwinkel $\left(^{\circ}\right)$ & $20,0 \pm 3,9$ & $20,1 \pm 4,7$ & $20,90 \pm 3,9$ \\
\hline ROM; Hüftgelenkswinkel $\left(^{\circ}\right)$ & $23,3 \pm 4,0$ & $20,7 \pm 3,1$ & $22,0 \pm 4,6$ \\
\hline ROM; Kniegelenkswinkel $\left(^{\circ}\right)$ & $34,4 \pm 10,8$ & $32,5 \pm 6,8$ & $29,6 \pm 6,4$ \\
\hline ROM; Sprunggelenkswinkel $\left(^{\circ}\right)$ & $30,9 \pm 6,6$ & $31,3 \pm 8,1$ & $29,1 \pm 5,9$ \\
\hline
\end{tabular}

Tabelle 4: Darstellung aller Schrittparameter der Gruppen N, MO, MA im natürlichen Gangbild: Signifikante Unterschiede zwischen Gruppen sind wie folgt gekennzeichnet: $N / M A={ }^{* *} p \leq 0,01, M O / M A={ }^{\circ} p \leq 0,05$;

Zunächst erfolgte eine Vergleichsanalyse der gesunden Probanden mit den beiden Migräne-Gruppen (siehe Tabelle 4). Im Verlauf wurden dann die Ergebnisse retrospektiv mit den Daten der Gruppe cerebellär erkrankter Patienten verglichen.

In der Analyse der Gangparameter der gesunden Patienten (N) im Vergleich zur Gruppe der Migräne-Patienten ohne Aura (MO) mittels Mann-Whitney-Test ergaben sich keine signifikanten Unterschiede. Die Probanden der MA-Gruppe wiesen jedoch eine signifikant kürzere Doppelstandphasendauer $(p=0,009)$ auf als die gesunden Probanden der Gruppe N. Ansonsten ließen sich ebenfalls keine relevanten Abweichungen ermitteln.

In der Analyse der beiden Migräne-Gruppen MO und MA mittels Mann-Whitney-Test konnte eine Signifikanz für die Stand- $(p=0,021)$ und Doppelstandphasendauer $(p=0,016)$ festgestellt werden. Dies lässt sich am ehesten durch die diskret divergierende Gehgeschwindigkeit in den beiden Gruppen erklären. Alle weiteren Gang-Parameter ergaben keine signifikanten Unterschiede, so dass im Folgenden beide Migräne-Gruppen zusammengefasst wurden ( $M O+M A=M)$.

Zum retrospektiven Vergleich der relativ gering divergierenden Ergebnisse der Gruppen N und M mit der Gruppe cerebellär Erkrankter (CD) wurde zunächst ein Kruskal-Wallis-Test durchgeführt. Dabei ergaben sich signifikante Auffälligkeiten bei der Gehgeschwindigkeit $(p=0,012)$, der Doppelstandphasendauer $(p=0,000)$ sowie dem Variationskoeffizient der Teilschrittlänge $(p=0,002)$, der Schritthöhe $(p=0,016)$ 
und der Doppelschrittlänge $(p=0,003)$. Als ebenfalls auffällig erwiesen sich die Schwung- $(p=0,010)$ und Standphasendauer $(p=0,012)$ sowie der jeweilige Variationskoeffizient von Schwung- $(p=0,000)$ und Standphase $(p=0,008)$.

Wie zu erwarten war bestätigten sich, mit Ausnahme der Standphasendauer und dem Schritthöhenvariationskoeffizienten, die meisten im Kruskal-Wallis-Test aufgetretenen Signifikanzen auch im Mann-Whitney-Test beim Vergleich der gesunden Probanden $(\mathrm{N})$ mit den Patienten mit cerebellärer Dysfunktion. Ähnliche Ergebnisse erbrachte der Mann-Whitney-Test zwischen der zusammengefassten Migräne-Gruppe $\mathrm{M}$ und den CD-Patienten. Alle bereits im Kruskal-Wallis-Test ermittelten, auffälligen Parameter wurden bei leicht divergierendem Niveau erneut als signifikant bestätigt. In der folgenden Tabelle wurden alle signifikanten Parameter dargestellt. 


\begin{tabular}{|c|c|c|c|}
\hline Schrittparameter & $\mathbf{N}$ & $\mathbf{M}$ & CD \\
\hline Gehgeschwindigkeit (m/s) & $1,19 \pm 0,15^{* \star}$ & $\begin{array}{c}1,23 \pm 0,11 \\
\text { ○。 }\end{array}$ & $0,89 \pm 0,32 * * \circ \circ$ \\
\hline $\begin{array}{l}\text { Variationskoeffizient der } \\
\text { Doppelschrittlänge (\%) }\end{array}$ & $2,4 \pm 2,5^{* *}$ & $\begin{array}{c}2,1 \pm 0,9 \\
\text { ○০० }\end{array}$ & $\begin{array}{c}4,9 \pm 1,9 \text { ** } \\
\text { ००० }\end{array}$ \\
\hline $\begin{array}{l}\text { Variationskoeffizient der } \\
\text { Teilschrittlänge (\%) }\end{array}$ & $3,4 \pm 1,8^{* \star *}$ & $\begin{array}{c}4,8 \pm 5,1 \\
\text { ○० }\end{array}$ & $\begin{array}{c}9,7 \pm 4,0 * * * \\
\circ \circ\end{array}$ \\
\hline Standphasendauer (ms) & $648,3 \pm 43,7$ & $\begin{array}{c}624,3 \pm 41,9 \\
\circ\end{array}$ & $\begin{array}{c}737,1 \pm 145,8 \\
\circ\end{array}$ \\
\hline $\begin{array}{l}\text { Variationskoeffizient der } \\
\text { Standphasendauer (\%) }\end{array}$ & $2,3 \pm 0,5^{* *}$ & $\begin{array}{c}2,6 \pm 1,1 \\
\text { ○० }\end{array}$ & $\begin{array}{c}4,2 \pm 1,7^{\star *} \\
\text { ॰ }\end{array}$ \\
\hline $\begin{array}{l}\text { Schwungphasendauer } \\
\text { (ms) }\end{array}$ & $408,5 \pm 15,3^{* *}$ & $\begin{array}{c}409,9 \pm 24,5 \\
\text { ○० }\end{array}$ & $\begin{array}{c}374,8 \pm 33,2^{* *} \\
\text { ○० }\end{array}$ \\
\hline $\begin{array}{c}\text { Variationskoeffizient der } \\
\text { Schwungphasendauer } \\
(\%)\end{array}$ & $2,1 \pm 0,6$ *** & $\begin{array}{c}2,7 \pm 1,2 \\
\text { ○०० }\end{array}$ & $\begin{array}{c}5,4 \pm 1,7^{\star * \star} \\
\text { ○०० }\end{array}$ \\
\hline $\begin{array}{l}\text { Doppelstandphasendauer } \\
\qquad(\mathrm{ms})\end{array}$ & $119,5 \pm 16,3^{* *}$ & $\begin{array}{c}107,9 \pm 12,4 \\
\text { ○०० }\end{array}$ & $\begin{array}{c}180,9 \pm 66,0 \text { ** } \\
\text { ○०० }\end{array}$ \\
\hline $\begin{array}{c}\text { Variationskoeffizient der } \\
\text { Schritthöhe (\%) }\end{array}$ & $4,5 \pm 3,3$ & $\begin{array}{c}3,8 \pm 3,9 \\
\text { ○० }\end{array}$ & $\begin{array}{c}6,3 \pm 2,7 \\
\text { ○о }\end{array}$ \\
\hline
\end{tabular}

Tabelle 5: Darstellung der Schrittparameter im natürlichen Gang im Vergleich von Normalprobanden, Migränepatienten und cerebellär Erkrankten.

Signifikante Unterschiede sind wie folgt gekennzeichnet:

$\mathrm{N} / \mathrm{CD}=\mathrm{p} \leq 0,05^{*} ; \mathrm{p} \leq 0,01^{* *} ; \mathrm{p} \leq 0,001^{* * *}$

$M(M O+M A) / C D=p \leq 0,05^{\circ} ; p \leq 0,01^{\circ} ; p \leq 0,001^{\circ 0 \circ}$ 

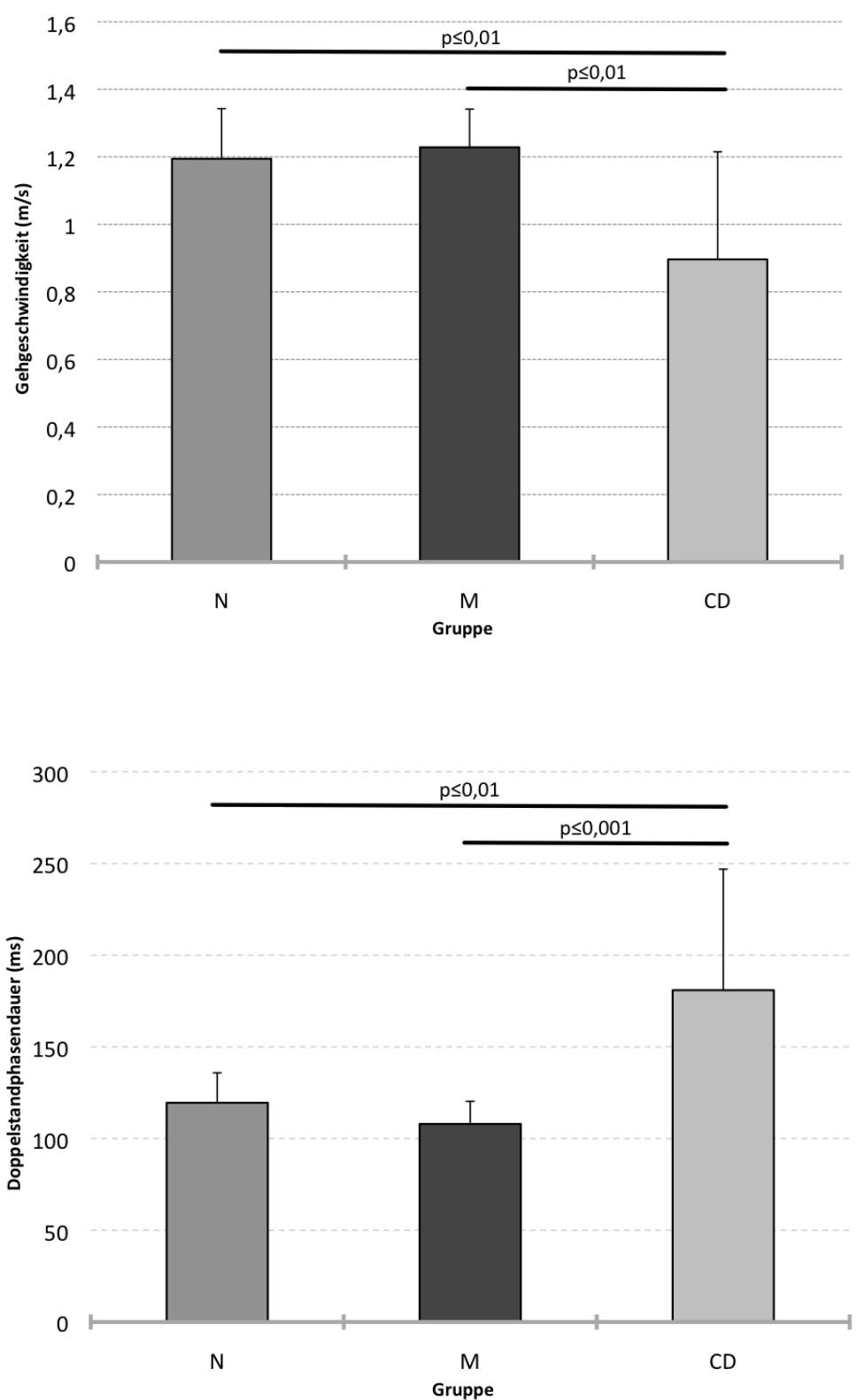

Abb. 4: Graphische Darstellung signifikant unterschiedlicher Parameter im natürlichen Gang gemäß der Ergebnisse aus Tabelle 5

\subsubsection{Korrelationen zu Gangparametern des natürlichen Gangbildes}

Wie bereits in der Analyse des Seiltänzerganges wurden die für den normalen Gang erhobenen Variablen auf relevante Korrelationen zu den anamnestisch erhobenen Migräne-Parametern untersucht. Aufgrund der geringen Unterschiede zwischen den Gruppen MO und MA wurden dabei die Daten aller 29 Migräne-Patienten, wie bereits erwähnt, zu einer Gruppe M zusammengefasst. 


\begin{tabular}{|c|c|c|}
\hline Klinische Daten & Schrittbreite & Doppelstandphasendauer \\
\hline Attackenfrequenz & 0,158 & $\mathbf{0 , 0 1 4}^{\star}$ \\
\hline Erkrankungsdauer & $\mathbf{0 , 0 1 8 ^ { \star }}$ & $\mathbf{0 , 0 6 2}$ \\
\hline Attackendauer & $\mathbf{0 , 5 5 9}$ & $\mathbf{0 , 0 0 8}^{\star \star}$ \\
\hline Kopfschmerzintensität & $\mathbf{0 , 3 9 8}$ & $\mathbf{0 , 0 1 1}^{\star}$ \\
\hline ICARS-Score & $0,002^{\star \star}$ & 0,201 \\
\hline
\end{tabular}

Tabelle 6: P-Werte der Korrelationen von klinischen Daten und Gangparametern im natürlichen Gang. Signifikante Korrelationen sind markiert: $p \leq 0,05$ *; $p \leq 0,01$ **

Wie in Tabelle 6 dargestellt ergaben sich signifikante Korrelationen der Erkrankungsdauer und der Schrittbreite sowie dem ICARS-Score und Schrittbreite $(p=0,002)$. Die übrigen Migräne-Parameter stellten sich diesbezüglich unauffällig dar. Des Weiteren lassen sich in der Analyse signifikante Korrelationen für die Attackenfrequenz, die Attackendauer sowie der subjektiven Kopfschmerzintensität zu der Doppelstandphasendauer ermitteln. Alle übrigen Parameter erwiesen sich im Pearson-Test als nicht signifikant korreliert.

\subsection{Seiltänzergang-Analyse}

\begin{tabular}{|c|c|c|c|}
\hline Schrittparameter & N & MO & MA \\
\hline Gehgeschwindigkeit (m/s) & $0,40 \pm 0,13$ & $0,38 \pm 0,11$ & $0,41 \pm 0,09$ \\
\hline Doppelschrittlänge (cm) & $66,0 \pm 14,4$ & $62,1 \pm 14,2$ & $67,0 \pm 12,1$ \\
\hline $\begin{array}{c}\text { Variationskoeffizient der } \\
\text { Doppelschrittlänge (\%) }\end{array}$ & $3,7 \pm 1,4$ & $5,1 \pm 1,8$ & $4,6 \pm 2,3$ \\
\hline Teilschrittlänge (cm) & $33,0 \pm 8,1$ & $31,4 \pm 7,5$ & $33,2 \pm 5,9$ \\
\hline $\begin{array}{c}\text { Variationskoeffizient der } \\
\text { Teilschrittlänge (\%) }\end{array}$ & $9,4 \pm 10,6$ & $9,8 \pm 8,9$ & $8,3 \pm 4,5$ \\
\hline Symmetriequotient (\%) & $1,0 \pm 0,1$ & $1,0 \pm 0,1$ & $1,0 \pm 0,6$ \\
\hline Standphasendauer (ms) & $1199,3 \pm 247,5$ & $1189,5 \pm 291,4$ & $1176,3 \pm 265,4$ \\
\hline Schwungphasendauer (ms) & $511,4 \pm 70,9$ & $477,0 \pm 55,3$ & $492,4 \pm 76,0$ \\
\hline
\end{tabular}




\begin{tabular}{|c|c|c|c|}
\hline $\begin{array}{l}\text { Doppelstandphasendauer } \\
\text { (ms) }\end{array}$ & $357,0 \pm 105,9$ & $352,9 \pm 123,0$ & $338,8 \pm 104,5$ \\
\hline Kadenz & $72,1 \pm 12,8$ & $74,1 \pm 13,4$ & $74,8 \pm 14,9$ \\
\hline Schrittbreite (mm) & $9,3 \pm 17,0$ & $17,8 \pm 16.0$ & $14,5 \pm 17,7$ \\
\hline $\begin{array}{l}\text { Variationskoeffizient der } \\
\text { Schrittbreite (\%) }\end{array}$ & $810,5 \pm 3170,0$ & $367,4 \pm 1026,4$ & $235,7 \pm 366,8$ \\
\hline Schritthöhe (mm) & $153,2 \pm 20,6$ * & $130,2 \pm 27,3^{*}$ & $140,0 \pm 29,5$ \\
\hline Fußwinkel $\left(^{\circ}\right)$ & $13,0 \pm 4,9$ & $11,8 \pm 3,8$ & $13,7 \pm 3,3$ \\
\hline $\begin{array}{l}\text { Varationskoeffizient des } \\
\text { Fußwinkels (\%) }\end{array}$ & $19,7 \pm 11,4$ & $21,0 \pm 7,6$ & $18,3 \pm 9,0$ \\
\hline ROM; Hüftgelenkswinkel $\left(^{\circ}\right)$ & $17,3 \pm 3,8^{*}$ & $14,3 \pm 2,5^{*}$ & $16,5 \pm 2,7$ \\
\hline ROM; Kniegelenkwinkel $\left({ }^{\circ}\right)$ & $\begin{array}{c}31,7 \pm 6,6 \text { * } \\
\text { ०० }\end{array}$ & $25,4 \pm 6,0$ * & $\begin{array}{c}23,6 \pm 8,7 \\
\text { ○о }\end{array}$ \\
\hline ROM; Sprunggelenkwinkel $\left(^{\circ}\right)$ & $23,2 \pm 4,3$ & $19,7 \pm 3,6$ & $20,4 \pm 6,6$ \\
\hline Ataxiequotient & $9,1 \pm 4,2^{\circ}$ & $8,6 \pm 3,4$ & $11,1 \pm 3,3^{\circ}$ \\
\hline Missteps & $\begin{array}{c}0,5 \pm 0,7^{* * *} \\
\text { ००० }\end{array}$ & $2,7 \pm 2,2^{* * *}$ & $\begin{array}{c}3,6 \pm 3,1 \\
\text { ○о० }\end{array}$ \\
\hline
\end{tabular}

Tabelle 7: Darstellung aller Schrittparameter der Gruppen N, MO, MA im Seiltänzergang: Signifikante Unterschiede sind wie folgt gekennzeichnet: $N / M O=p \leq 0,05$ *; $p \leq 0,01^{* *} ; p \leq 0,001^{* * *} ; N / M A=p \leq 0,05^{\circ} ; p \leq 0,01^{\circ \circ} ; p \leq 0,001^{\circ \circ}$;

Bei der Untersuchung im Seiltänzergang fanden sich im Vergleich der gesunden Probanden mit Migräne-Patienten beider Gruppen signifikante Unterschiede. Die MO-Patienten hatten im Mann-Whitney-Test gegenüber der Gruppe N eine auffallend niedrigere Schritthöhe $(p=0,014)$, vermehrt Fehltritte $(p=0,000)$ sowie auffällige Hüft$(p=0,016)$ und Kniegelenkswinkel $(p=0,039)$. Die Migräne-Patienten mit Aura (MA) wiesen neben den vermehrten Fehltritten $(p=0,000)$ und dem niedrigen Bewegungsumfang im Kniegelenkswinkel $(p=0,002)$ zusätzlich noch einen erhöhten Ataxie-Quotient auf $(p=0,038)$.

Beim Vergleich der beiden Migräne-Gruppen untereinander (MO/MA) ließen sich keine signifikanten Unterschiede berechnen. Für den retrospektiven Vergleich mit den Daten der cerebellär erkrankten Probanden wurden daher erneut beide MigräneGruppen zu einer gemeinsamen Gruppe zusammengefasst ( $\mathrm{MO}+\mathrm{MA}=\mathrm{M})$. 

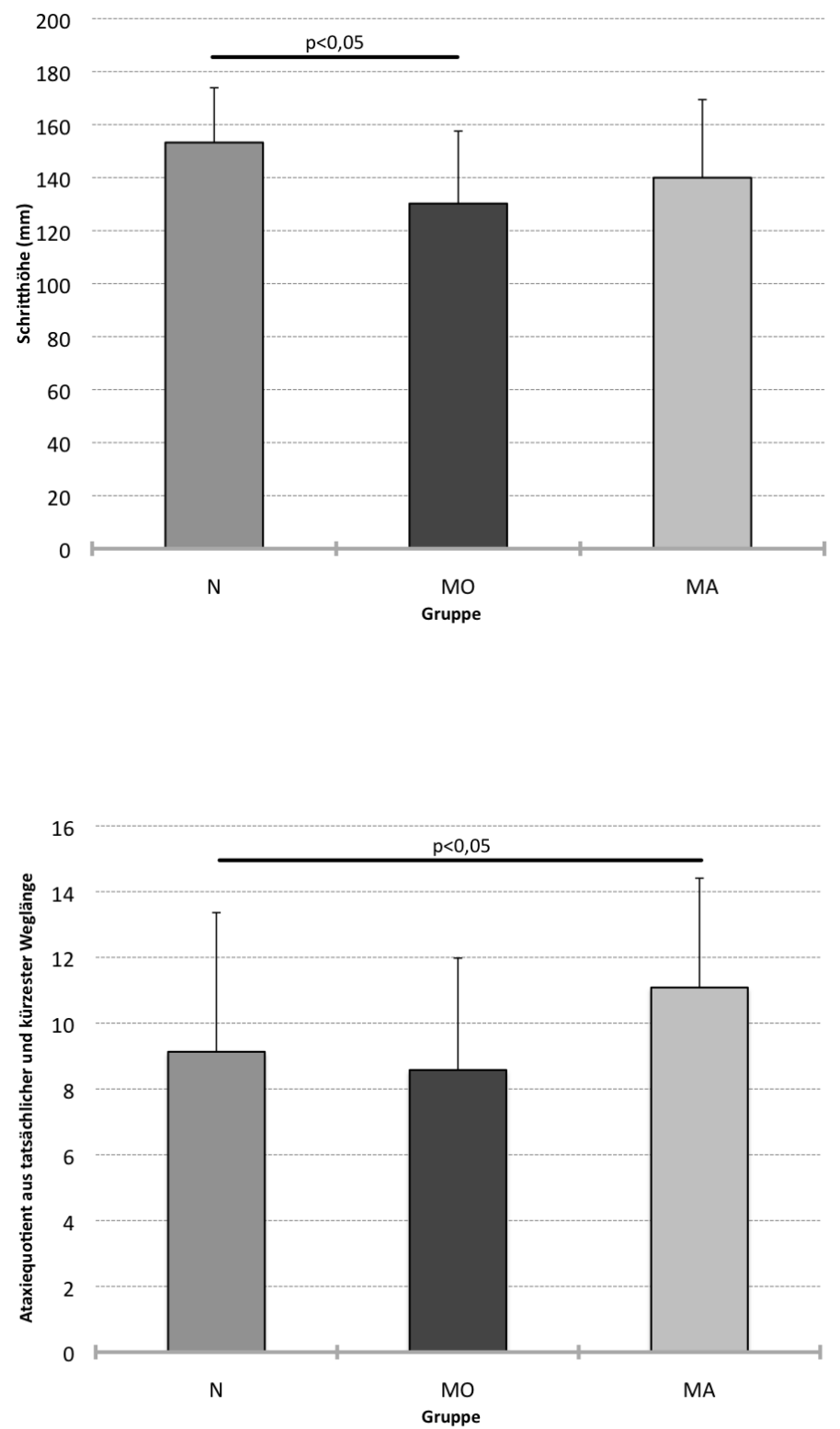

Abb. 5: Graphische Darstellung signifikant unterschiedlicher Parameter der SeiltänzergangAnalyse gemäß der Ergebnisse aus Tabelle 7 


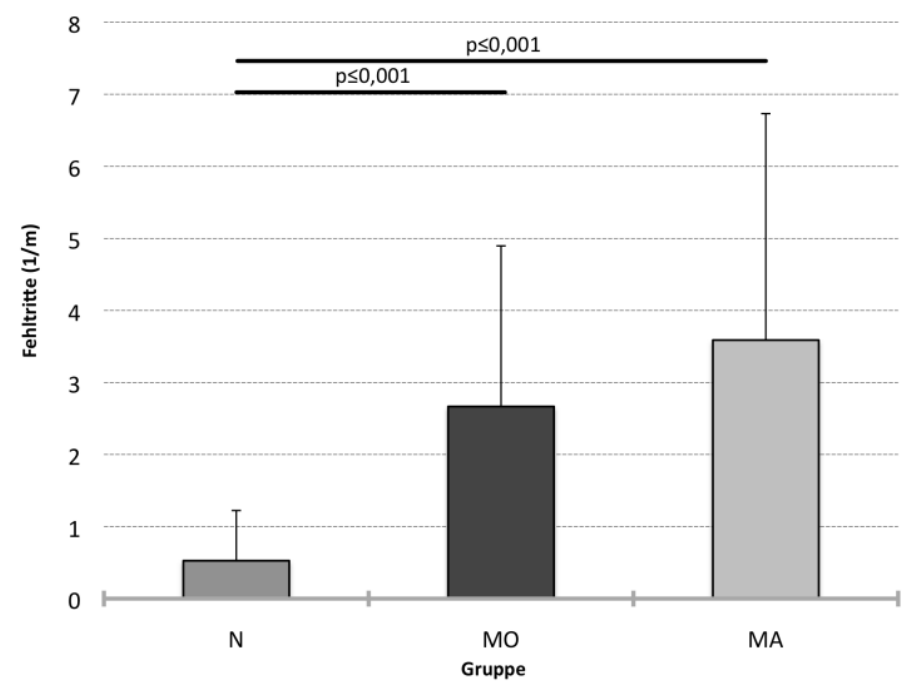

Abb. 6: Graphische Darstellung signifikant unterschiedlicher Parameter der SeiltänzergangAnalyse gemäß der Ergebnisse aus Tabelle 7, 2.Teil

In der Analyse der drei Gruppen (N; M; CD) ergaben sich mittels Kruskal-Wallis-Test signifikante Unterschiede in der Doppelstandphasendauer $(p=0,003)$, der Schritthöhe $(p=0,000)$, den Fehltritten $(p=0,000)$ sowie der Kniegelenkswinkel $(p=0,001)$. Ebenfalls auffällig war der ermittelte Ataxie-Quotient mit einem berechneten Signifikanzniveau von $\mathrm{p}=0,001$.

Im Mann-Whitney-Test wurden diese Auffälligkeiten beim Vergleich der Normalprobanden mit den retrospektiven Daten der untersuchten Patienten mit cerebellärer Dysfunktion bestätigt. Die CD-Probanden wiesen somit eine längere Doppelstandphase $(p=0,002)$, eine niedrige Schritthöhe $(p=0,000)$, eine erhöhte Anzahl an Fehltritten pro Minute $(p=0,000)$ sowie eine verlängerte Wegstrecke (Ataxie-Quotient) mit einem Signifikanzniveau von $\mathrm{p}=0,000$ auf. Zur Beurteilung der Winkelmaße der cerebellär erkrankten Patienten lagen keine Daten vor.

In der Analyse der Gruppe M verglichen mit der Gruppe CD wurden vergleichbar hohe Signifikanzen ermittelt (Doppelstandphasendauer $p=0,001$; Schritthöhe $p=0,007$; Ataxie-Quotient $p=0,007)$. Auffallend war jedoch ein nicht signifikanter Unterschied zwischen den CD-Patienten und den Probanden der Migräne-Gruppe mit einem Niveau von $p=0,236$ bezüglich der Fehltritte. Demnach unterliefen den cerebellär Erkrankten nicht signifikant mehr Fehltritte im Seiltänzergang als den 
Migräne-Patienten. Beide Gruppen unterschieden sich jedoch, wie bereits beschrieben, signifikant von der Vergleichsgruppe der gesunden Probanden.

\begin{tabular}{|c|c|c|c|}
\hline Schrittparameter & $\mathbf{N}$ & $\mathbf{M}$ & CD \\
\hline $\begin{array}{l}\text { Doppelstandphasendauer } \\
\text { (ms) }\end{array}$ & $357,0 \pm 105,9$ *** & $\begin{array}{c}344,6 \pm 110,6 \\
\text { о०० }\end{array}$ & $\begin{array}{c}604,1 \pm 251,2^{* * *} \\
\text { ○०० }\end{array}$ \\
\hline Schritthöhe (mm) & $153,2 \pm 20,6^{* * *}$ & $\begin{array}{c}135,9 \pm 28,5 \\
\text { o० }\end{array}$ & $\begin{array}{c}105,9 \pm 20,2^{* * *} \\
\text { ०० }\end{array}$ \\
\hline Ataxiequotient & $9,1 \pm 4,2^{* \star \star}$ & $\begin{array}{c}10,0 \pm 3,5 \\
\text { ○০० }\end{array}$ & $\begin{array}{c}22,8 \pm 11,1^{* \star *} \\
\text { ००० }\end{array}$ \\
\hline Missteps & $\begin{array}{c}0,5 \pm 0,7^{* * *} \\
\wedge \wedge \wedge\end{array}$ & $\begin{array}{c}3,2 \pm 2,8 \\
\wedge \wedge \wedge\end{array}$ & $9,1 \pm 12,7^{\star \star \star}$ \\
\hline
\end{tabular}

Tabelle 8: Signifikant unterschiedliche Schrittparameter im Seiltänzergang im Vergleich; $\mathrm{N} / \mathrm{CD}={ }^{* \star *}$ wenn $\mathrm{p} \leq 0,001 ; \mathrm{M} / \mathrm{CD}={ }^{\circ}$ wenn $\mathrm{p} \leq 0,01$; ${ }^{\circ}$ wenn $\mathrm{p} \leq 0,001$; $N / M=\wedge \wedge \wedge$ wenn $p \leq 0,001$

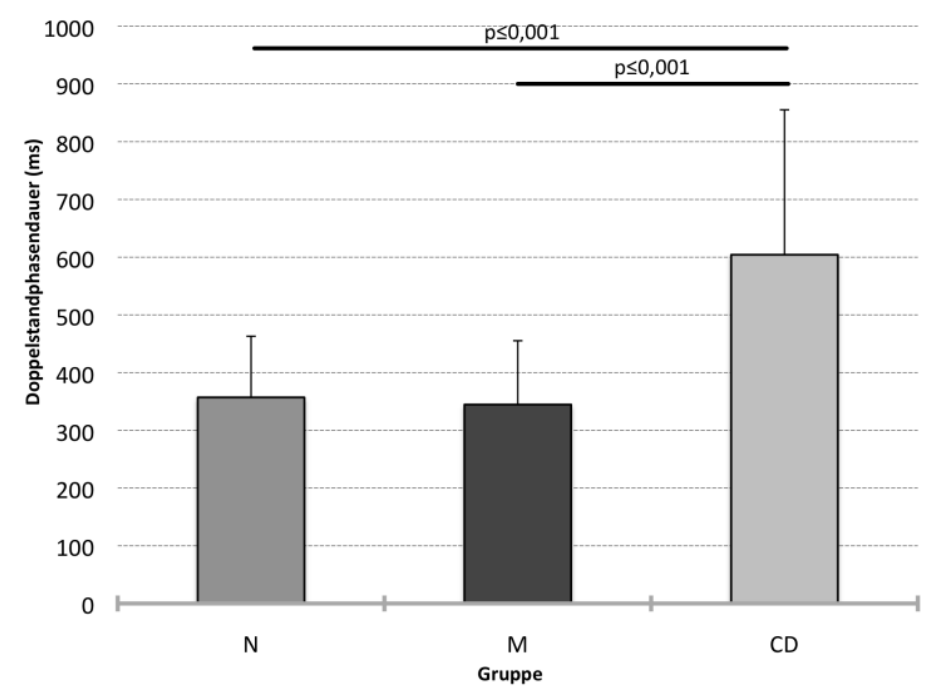

Abb. 7: Graphische Darstellung signifikant unterschiedlicher Parameter der SeiltänzergangAnalyse (N, M, CD) gemäß der Ergebnisse aus Tabelle 8 

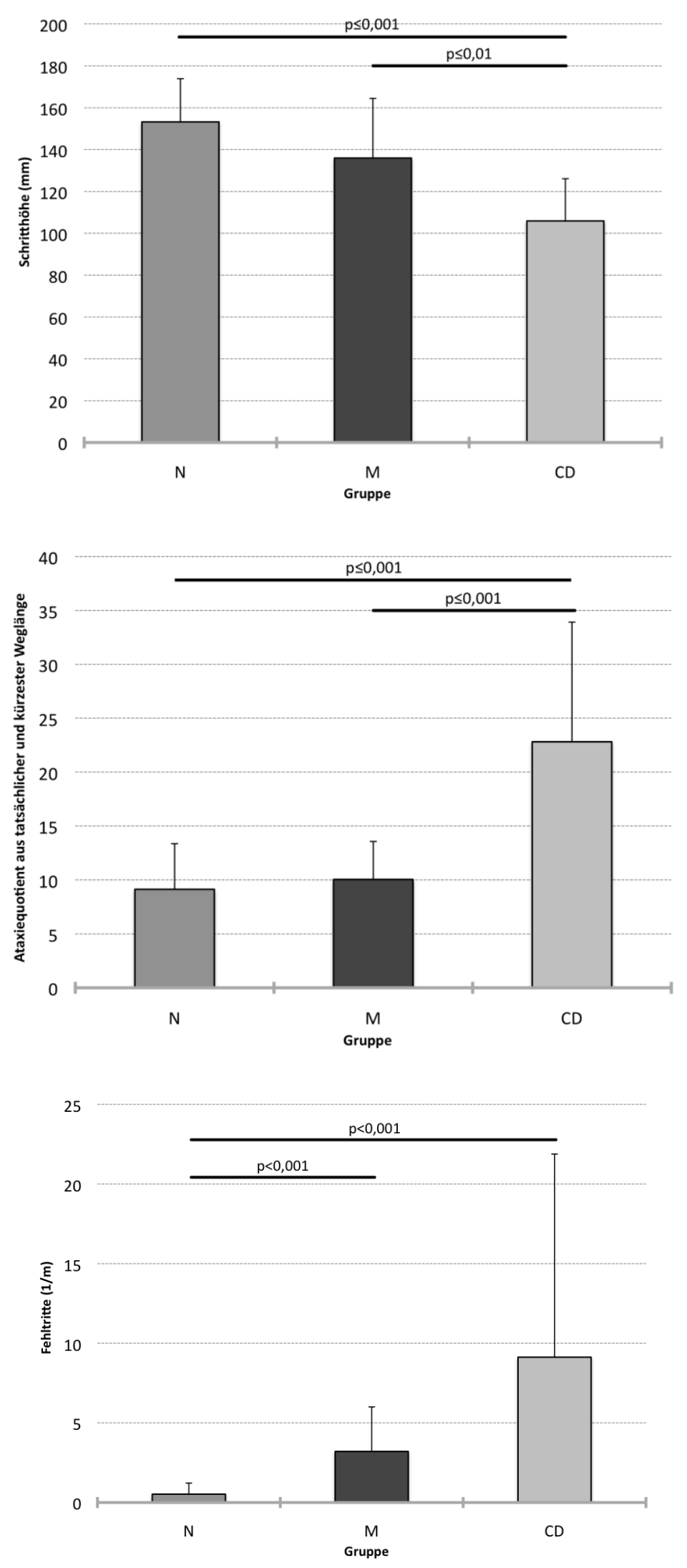

Abb. 8: Graphische Darstellung signifikant unterschiedlicher Parameter der SeiltänzergangAnalyse (N,M,CD) gemäß der Ergebnisse aus Tabelle 8, 2.Teil 

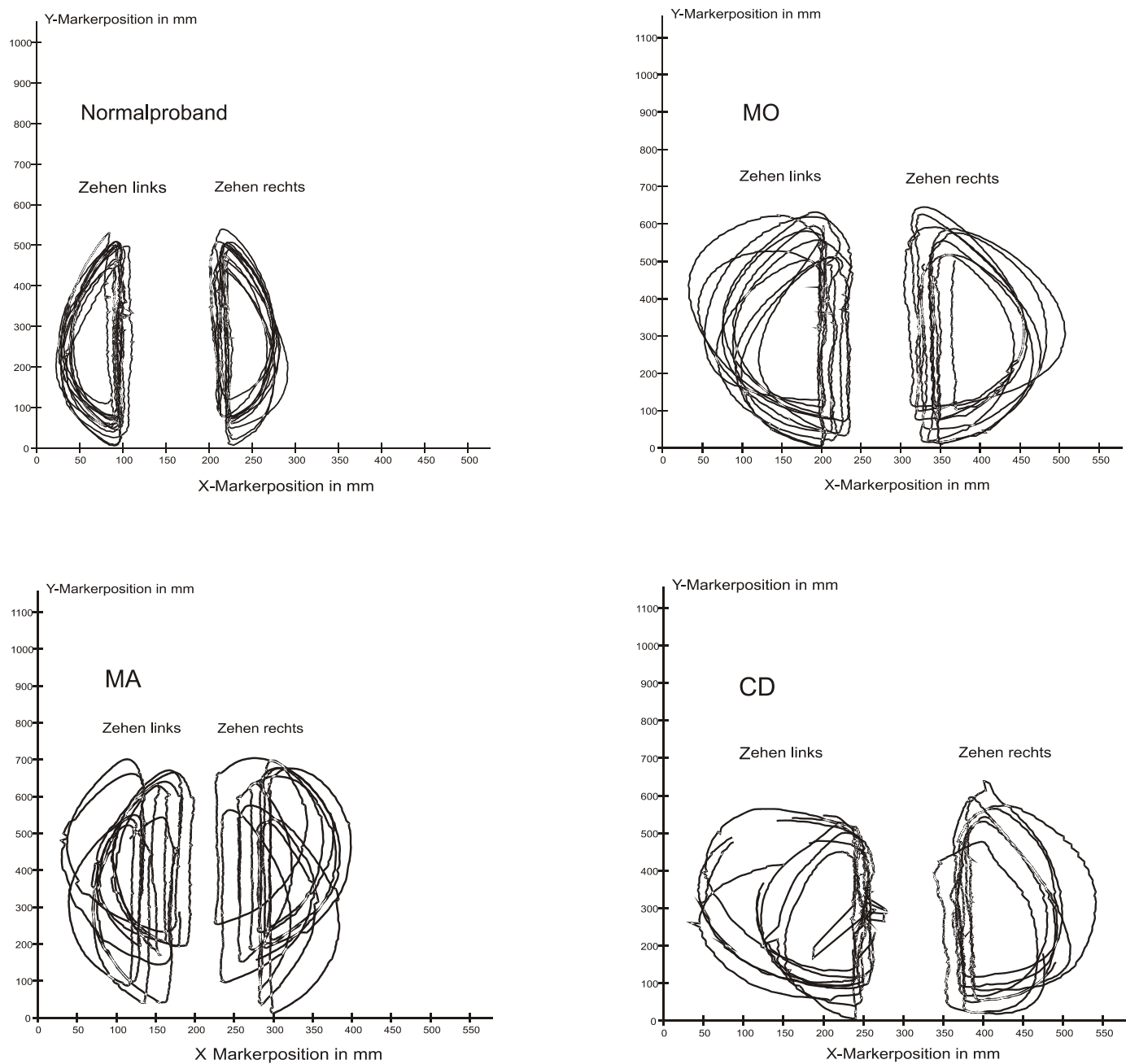

Abb. 9: Graphische Darstellung der Zehenmarker als Pendelkurve in der SeiltänzergangAnalyse in der horizontalen Ebene einer 20 sekündigen Laufbandmessung. Auf der x-Achse sind senkrecht zur Gangrichtung die Abweichungen der optimalen Wegstrecke abgebildet. Der y-Achse ist die Wegstrecke in Gangrichtung zu entnehmen. 


\subsubsection{Korrelationen zu Gangparametern des Seiltänzerganges}

Zur Auswertung relevanter Korrelationen zwischen Parametern der MigräneErkrankung und koordinativ aussagekräftigen Variablen der Ganganalyse wurden die Daten aller 29 untersuchten Migräne-Patienten zusammen betrachtet.

\begin{tabular}{|c|c|c|c|c|}
\hline Klinische Daten & ICARS-Score & Schrittbreite & Ataxie-Quotient & Missteps \\
\hline Attackenfrequenz & $0,004^{\star *}$ & 0,112 & 0,110 & $0,000^{\star \star *}$ \\
\hline Erkrankungsdauer & $0,000^{\star \star *}$ & 0,272 & $0,007^{\star \star *}$ & $0,000^{\star \star \star}$ \\
\hline Attackendauer & $0,021^{\star}$ & 0,198 & 0,421 & $0,000^{\star \star *}$ \\
\hline Intensität & $0,013^{*}$ & 0,076 & 0,219 & $0,000^{\star \star *}$ \\
\hline
\end{tabular}

Tabelle 9: P-Werte der Korrelationen von klinischen Daten und Gangparametern im Seiltänzergang. Korrelationen mit einem Signifikanzniveau von $p \leq 0,05\left({ }^{*}\right)$, $\left.p \leq 0,01{ }^{* *}\right)$ sowie $\left.p \leq 0,001{ }^{* * *}\right)$ sind markiert.

Korrelationen zu den anamnestisch erhobenen Migräneparametern lassen sich, wie in Tabelle 9 dargestellt, in allen Fällen mit dem ermittelten ICARS-Score und den Fehltritten feststellen. Auffällig ist dabei das hohe Signifikanzniveau für alle Parameter in Bezug auf die Fehltritte. Zusätzlich besteht in Bezug auf die Erkrankungsdauer noch eine Korrelation zum Ataxie-Quotient, die bei den übrigen Migräne-Parametern unauffällig ausfällt. In der Betrachtung der Schrittbreite ergeben sich keinerlei signifikante Korrelationen. 


\section{Diskussion}

\subsection{Cerebelläre Gangstörung bei Migräne-Patienten?}

Ziel der vorliegenden Studie war es zu überprüfen, ob sich im Vergleich von Menschen ohne Migräne mit Migränepatienten Auffälligkeiten in den Bewegungsabläufen finden, die eine Störung des Cerebellums vermuten lassen. Da die Gangmotorik eine elementare Funktion darstellt und eine Störung bereits für Zielbewegungen der Hände gezeigt wurde (Sandor et al. 2001), wählten wir ein entsprechendes Gangparadigma aus.

In Betrachtung der Ergebnisse der Seiltänzergang-Analyse imponiert eine signifikant erhöhte Anzahl an Fehltritten in beiden Migräne-Gruppen gegenüber den gesunden Probanden der Kontroll-Gruppe. Im direkten Vergleich besteht diesbezüglich kein signifikanter Unterschied zwischen Migränepatienten mit Aura und solchen ohne Aura. Unterschiede finden sich jedoch zu den Normalprobanden. So ist die Schritthöhe der Migräne-Patienten signifikant erniedrigt. Bezüglich des AtaxieQuotienten stellt sich eine signifikant erhöhte Abweichung der optimalen Wegstrecke der Migränepatienten während des Schrittsetzens dar.

Auffällige, cerebelläre Befunde wie diese wurden bereits in früheren Arbeiten beschrieben (Ambrosini et al., 2008; Harno et al., 2003) und unterstreichen die These der cerebellären Beteiligung bezüglich der weiterhin unklaren Pathophysiologie der Migräne-Erkrankung. Ambrosini et al. konnten des Weiteren Auffälligkeiten bezüglich der neuromuskulären Übertragung zusammen mit cerebellären Funktionen bei Migränepatienten mit Aura darstellen (Ambrosini et al., 2008). Als unterstützendes Ergebnis für die Hypothese, dass das Cerebellum in der Pathophysiologie der Migräne eine Rolle spielt, muss der Fakt angesehen werden, dass sowohl bei Migränepatienten als auch bei den cerebellär erkrankten Probanden die Anzahl der Fehltritte erhöht ist.

1993 wurden von Russell et al. in Cephalalgia eine genetische, am ehesten multifaktorielle Genese der Migräne-Erkrankung beschrieben. Dieser Ansatz galt sowohl für Migräne mit - als auch ohne Aura und wurde in weiteren Publikationen bestätigt (vergleiche Terwindt et al. 2001). Erste evidente Untersuchungen beschrieben eine langsam progressive Ataxie bei Patienten mit familiär hemiplegischer Migräne (FHM) (Russell et al. 1993). In der Folgezeit konnten in 
genetischen Tests und Untersuchungen verschiedene Mutationen der alpha 1AUntereinheit des P/Q Calcium-Kanals des Gehirns ermittelt werden (Alonso et al. 2003; Friend et al. 1999). Laut einer Publikation von Terwindt et al. 2001 in Neurology sei bei ungefähr 50\% der untersuchten Familien eine diagnostizierte FHM auf Mutationen des beschriebenen CACNA1A-Genlokus auf dem Chromosom 19p13 zurückzuführen. Diese Mutation konnte neben der FHM auch bei der episodischen Ataxie Typ 2 sowie bei der spinocerebellären Ataxie Typ 6 nachgewiesen werden (Alonso et al. 2003).

Konkrete Hinweise für genetisch determinierte Mutationen wie bei der FHM ließen sich für die Migräne mit - und ohne Aura bislang nicht ermitteln. Ferner wird eine polygenetische multifaktorielle Genese der Erkrankung angenommen (Ducros et al. 2002). Dennoch weisen die Ergebnisse dieser Studie mit signifikanten Auffälligkeiten beider Formen der Migräne in koordinativen Testungen, ebenso wie die Resultate von Sandór et al. 2001 und Harno et al. 2003, auf eine mögliche cerebelläre Komponente hin.

Bemerkenswert ist der Vergleich der Ergebnisse dieser beiden Publikationen mit den Ergebnissen der vorliegenden Studie. Sandór et al. (2001) beschreiben in ihrem Zeigeversuch-Paradigma das Auftreten cerebellärer Symptome in beiden Formen der Migräne, jedoch eine eindeutige Betonung der Symptome bei den Migräneprobanden mit Aura. Ähnliche Ergebnisse lassen sich der Publikation von Harno et al. (2003) entnehmen. Dort werden ebenfalls abnormale Auffälligkeiten in umfassenden neurologischen Testungen sowohl bei Migränepatienten mit - als auch ohne Aura beschrieben. Auch in diesen Ergebnissen seien die Befunde der Migränepatienten mit Aura deutlich auffälliger. In der vorliegenden Studie kam es, wie bereits beschrieben, zu signifikant koordinativ schlechteren Ergebnissen der Migräneprobanden im Vergleich zur Gruppe der Gesunden. Vor allem in Bezug auf die Ergebnisse der Fehltritte im Seiltänzergang sowie der verlängerten Wegstrecke im Ataxie-Quotient erzielen die Migränepatienten mit Aura schlechtere Ergebnisse als die Probanden mit Migräne ohne Aura.

Diese These findet Unterstützung in der Betrachtung der in Kapitel 3.2 beschriebenen Ergebnisse der koordinativen Testung gemäß dem Ataxie-Score von Trouillas et al. (1997). In der Summe der bewerteten Übungen erzielen die Probanden der Gruppe MA signifikant schlechtere Ergebnisse als die Gesunden. Den größten Teil machten dabei Auffälligkeiten in der Untergruppe Haltung und 
Gang aus, wie z.B. ein verbreiteter Fußabstand beim sicheren, selbst gewählten Stand. Deutlich geringer war der Anteil von Störungen der Kinetik, wie z.B. der Diadochokinese sowie der artikulatorischen- und okulären Motorik.

Bei Probanden der Gruppe MO waren ebenfalls koordinative Defizite zu eruieren. Jedoch erreichte der Unterschied zur Vergleichsgruppe N keine Signifikanz. Diese Tendenz zeigt eine erneute Kongruenz in den Ergebnissen dieser Studie mit denen von Sandor et al. 2001 sowie von Harno et al. 2003.

Trotz einer fehlenden genetischen Determinierung lassen sich folglich in Zusammenschau aller drei Studien reproduzierbar cerebelläre Defizite bei Migränepatienten ermitteln, die eine größere Ausprägung bei Patienten mit Aura aufweisen. Dennoch würden wir als Untersucher in Zusammenschau der aktuellen Befunde und Ergebnisse eher von einer koordinativen Störung bei Migränepatienten sprechen, da eine rein cerebelläre Genese nicht ausreichend belegt werden konnte.

\subsection{Korrelation von Migräne-Variablen und Gangparametern}

In der Analyse bezüglich einer möglichen Korrelation zwischen Variablen der Migräne-Erkrankung sowie spezifischen Parametern der Ganganalyse, wurden alle erhobenen Daten (MO+MA; $n=29)$ zu einer gemeinsamen Gruppe zusammengefasst.

Bei der Betrachtung der Ergebnisse imponiert eine signifikante Korrelation zwischen der ICARS-Skala (Trouillas et al. 1997) und den krankheitsspezifischen Daten der Migränepatienten. Am eindeutigsten fällt das Ergebnis bezüglich der ermittelten Fehltritte aus. Hier zeigt sich für alle Migräne-Parameter eine signifikant hohe Übereinstimmung (siehe Kapitel 3.4.1). Folglich scheint die Ausprägung der beschriebenen koordinativen Defizite in hohem Maße von der Erkrankungsdauer, Attackendauer, Attackenfrequenz und Intensität der Migräne-Erkrankung abhängig zu sein. Eine Objektivierung dieser These erscheint jedoch durchaus schwierig, da sich die ermittelten Migräne-Parameter ausschließlich auf anamnestisch erhobene Aussagen der Probanden stützen. An diesem Punkt offenbart sich die Schwierigkeit in der Erforschung der Migräne-Erkrankung, objektivierbare Parameter zu bestimmen, welche verlässliche Aussagen über die Pathophysiologie zulassen. Dennoch ist das hohe Signifikanzniveau aller Parameter in dieser Korrelation als auffällig zu bewerten. 
Auch in der Analyse des natürlichen Gangbildes ergaben sich interessante Korrelationen zwischen der Erkrankungsdauer sowie der erreichten Punkte im ICARS-Score (Trouillas et al. 1997) der Patienten mit der Schrittbreite beim Gehen. Patienten mit einem langen Erkrankungszeitraum weisen demnach eine erhöhte Schrittbreite auf, was für eine alterierte Gleichgewichtsstörung spricht. Die Schrittbreite war außerdem bei den Patienten mit einem koordinativ schlechten Ergebnis im ICARS-Score deutlich erhöht. Dass ein Patient mit einem hohen Score, also einem schlechten Ergebnis in der koordinativen Testung, in der weiteren Prüfung des Gangbildes auch eine größere Schrittbreite als koordinativ unauffällige Probanden aufweist, ist zu erwarten gewesen. Das hohe Signifikanzniveau in der Korrelation sowie die Kongruenz der einzelnen Ergebnisse beider Testverfahren (Bewertung der Koordination mittels Trouillas-Ataxie-Skala sowie der computergestützten Ganganalyse) unterstreicht jedoch erneut die Theorie der cerebellären Beteiligung in der Pathophysiologie der Migräne-Erkrankung und spiegelt die Qualität der verwendeten Untersuchungsmethoden wieder.

Stolze beschrieb 1995 in einer klinischen Studie zum Gangbild im Alter und beim Morbus Parkinson die Funktion der Schrittbreite beim Gehen als maßgeblichen Faktor zur Aufrechterhaltung des Gleichgewichtes und damit zur dynamischen Haltungsstabilität (vergleiche Guimares und Isaacs 1980; Hirokawa 1989). Ein nennenswerter Unterschied in der Schrittbreite der untersuchten Probanden verschiedener Altersklassen lies sich nicht ermitteln, so dass eine generelle Erhöhung der Schrittbreite im höheren Lebensalter nicht anzunehmen ist. Folglich ist die Korrelation der vorliegenden Studie von Erkrankungsdauer und Erhöhung der Schrittbreite nicht durch das erhöhte Lebensalter, sondern durch die koordinativen Defizite der Migräne-Erkrankung zu erklären. Warum es jedoch zu einer Zunahme der Schrittbreite im weiteren Krankheitsverlauf kommt, bleibt unklar. 


\section{Zusammenfassung}

Ziel dieser klinischen Studie war es, Migräne-Patienten mit und ohne Aura im attackenfreien Intervall einer Ganganalyse zu unterziehen, um Hinweise auf eine mögliche cerebelläre Beteiligung bezüglich der pathophysiologischen Genese der Migräne-Erkrankung zu erhalten. Zu diesem Zweck wurden die Ergebnisse der Ganganalyse mit denen gesunder Normalprobanden sowie cerebellär erkrankter Patienten verglichen. Essentiell zur komplikationslosen Durchführung waren die Einteilung der rekrutierten Probanden in die entsprechende Migräne-Diagnose gemäß der Klassifikation der International Headache Society sowie die Einhaltung der definierten Ausschlusskriterien.

Der methodische Aufbau basierte auf der Bestimmung eines standardisierten AtaxieScores, welcher von Trouillas et al. 1997 entwickelt und publiziert wurde sowie auf einer standardisierten Ganganalyse mittels ProReflex® System. Dabei wurden, nach Ermittlung der individuellen Gehgeschwindigkeit, eine Vielzahl von relevanten Parametern durch das Gehen auf einem Laufband im natürlichen Gang und im Seiltänzergang bestimmt. Als wesentliche Variablen erwiesen sich die Fehltritte im Seiltänzergang, die Schritthöhe, die Schrittbreite sowie der Ataxie-Quotient.

Die vorliegende Studie zeigt, dass Migräne-Patienten signifikant schlechtere Ergebnisse im Seiltänzergang gegenüber gesunden Vergleichsprobanden erreichen. Das Ausmaß dieser Abweichungen ist jedoch als diskret zu bezeichnen. Betrachtet man retrospektiv die Ergebnisse cerebellär erkrankter Patienten im Vergleich, so fällt eine weit aus deutlichere Abweichung zu den Werten der gesunden Probanden auf. Des Weiteren ergibt die Auswertung Unterschiede zwischen den beiden MigräneGruppen MO und MA. Migräne-Patienten mit Aura erzielen demnach schlechtere koordinative Resultate, besonders in Bezug auf den Ataxie-Quotienten, welcher ähnlich dem der cerebellären Patienten war. Vergleichbare Ergebnisse erbrachten bereits die Publikationen von Sandor et al. 2001 und Harno et al. 2003 für die obere Extremität. Des Weiteren scheint die Ausprägung der beschriebenen koordinativen Defizite in hohem Maße von der Erkrankungsdauer, Attackendauer, Attackenfrequenz und Intensität der Migräne-Erkrankung abhängig zu sein.

In Zusammenschau der Ergebnisse der vorliegenden Arbeit konnten weitere Hinweise auf eine cerebelläre Mitbeteiligung an der Pathophysiologie der MigräneErkrankung ermittelt werden. Eine Übereinstimmung mit Resultaten vergleichbarer 
Publikationen der letzten Jahre bestärkt diese These. In welchem Maße Genanalysen oder hier beschriebene koordinative Testungen zukünftig eine Relevanz bezüglich der Diagnostik und Therapie darstellen werden, bleibt abzuwarten. 


\section{Literaturverzeichnis}

Adams PJ, Snutch TP. Calcium channelopathies: voltage-gated calcium channels. Subcell Biochem. 2007;45:215-51.

Alonso I, Barros J, Tuna A, Coelho J, Sequeiros J, Silveira I, Coutinho P. Phenotypes of spinocerebellar ataxia type 6 and familial hemiplegic migraine caused by a unique CACNA1A missense mutation in patients from a large family. Arch Neurol. 2003 Apr;60(4):610-4.

Ambrosini A, Pierelli F, Schoenen J. Acetazolamide acts on neuromuscular transmission abnormalities found in some migraineurs. Cephalalgia. 2003 Mar;23(2):75-8.

Ambrosini A, de Noordhout AM, Alagona G, Dalpozzo F, Schoenen J. Impairment of neuromuscular transmission in a subgroup of migraine patients. Neurosci Lett. 1999 Dec 10;276(3):201-3.

Ambrosini A, Sándor PS, De Pasqua V, Pierelli F, Schoenen J. Performances in cerebellar and neuromuscular transmission tests are correlated in migraine with aura. J Headache Pain. 2008 Feb;9(1):29-32. Epub 2008 Feb 5.

Ambrosini A, Schoenen J. Electrophysiological response patterns of primary sensory cortices in migraine. J Headache Pain. 2006 Dec;7(6):377-88. Epub 2006 Dec 13.

Bawa B, Abbott LC. Analysis of calcium ion homeostasis and mitochondrial function in cerebellar granule cells of adult $\mathrm{CaV} 2.1$ calcium ion channel mutant mice. Neurotox Res. 2008 Jan;13(1):1-18. 
Battistini S, Stenirri S, Piatti M, Gelfi C, Righetti PG, Rocchi R, Giannini F, Battistini N, Guazzi GC, Ferrari M, Carrera P. A new CACNA1A gene mutation in acetazolamide-responsive familial hemiplegic migraine and ataxia. Neurology. 1999 Jul 13;53(1):3-4.

Benatar M, Ford CC. Familial hemiplegic migraine: more than just a headache. Neurology. 2005 Feb 22;64(4):592-3.

Carrera $\mathrm{P}$, Stenirri S, Ferrari M, Battistini S. Familial hemiplegic migraine: a ion channel disorder. Brain Res Bull. 2001 Oct-Nov 1;56(3-4):239-41.

Catterall WA, Few AP. Calcium channel regulation and presynaptic plasticity. Neuron. 2008 Sep 25;59(6):882-901.

Coban A, Baslo MB, Baykan B, Tutkavul K, Orhan EK, Ertas M. Subclinical neuromuscular transmission abnormality detected by single-fibre EMG is more pronounced in cluster headache than in migraine with aura. Cephalalgia. 2007 Jul;27(7):788-92.

Cricchi F, Di Lorenzo C, Grieco GS, Rengo C, Cardinale A, Racaniello M, Santorelli FM, Nappi G, Pierelli F, Casali C Early-onset progressive ataxia associated with the first CACNA1A mutation identified within the I-II loop. J Neurol Sci. 2007 Mar 15; 254(1-2):69-71. Epub 2007 Feb 12.

De Fusco M, Marconi R, Silvestri L, Atorino L, Rampoldi L, Morgante L, Ballabio A, Aridon P, Casari G. Haploinsufficiency of ATP1A2 encoding the $\mathrm{Na}+\mathrm{K}+$ pump alpha2 subunit associated with familial hemiplegic migraine type 2. Nat Genet. 2003 Feb;33(2):192-6. Epub 2003 Jan 21. 
Dichgans M, Herzog J, Freilinger T, Wilke M, Auer DP. 1H-MRS alterations in the cerebellum of patients with familial hemiplegic migraine type 1. Neurology. 2005 Feb 22;64(4):592-3.

Domitrz I, Kostera-Pruszczyk A, Kwieciñski H. A single-fibre EMG study of neuromuscular transmission in migraine patients. Cephalalgia. 2005 Oct; 25(10):81721.

Ducros A. Familial and sporadic hemiplegic migraine. Rev Neurol (Paris). 2008 Mar; 164(3):216-24. Epub 2008 Mar 11.

Ducros A, Tournier-Lasserve E, Bousser MG. The genetics of migraine. Lancet Neurol. 2002 Sep; 1(5):285-93.

Felix R Calcium channelopathies. Neuromolecular Med. 2006; 8(3):307-18.

Freilinger T, Dichgans M. Genetics of migraine. Nervenarzt. 2006 Oct; 77(10):1186, 1188-95.

Friend KL, Crimmins D, Phan TG, Sue CM, Colley A, Fung VS, Morris JG, Sutherland GR, Richards RI. Detection of a novel missense mutation and second recurrent mutation in the CACNA1A gene in individuals with EA-2 and FHM. Hum Genet. 1999 Sep;105(3):261-5.

Frontali M. Spinocerebellar ataxia type 6: channelopathy or glutamine repeat disorder? Brain Res Bull. 2001 Oct-Nov 1; 56(3-4):227-31.

Gordon N. Episodic ataxia and channelopathies. Brain Dev. 1998 Jan; 20(1):9-13. 
Guimaraes RM, Isaacs B. Characteristics of the gait in old people who fall. Int Rehabil Med. 1980; 2(4):177-80.

Haan J, Kors EE, Vanmolkot KR, van den Maagdenberg AM, Frants RR, Ferrari MD. Migraine genetics: an update. Curr Pain Headache Rep. 2005 Jun; 9(3):213-20.

Haan J, Terwindt GM, Ferrari MD. Genetics of migraine. Neurol Clin. 1997 Feb;15(1):43-60.

Harno H, Heikkinen S, Kaunisto MA, Kallela M, Häkkinen AM, Wessman M, Färkkilä M, Lundbom N. Decreased cerebellar total creatine in episodic ataxia type 2: a $1 \mathrm{H}$ MRS study. Neurology. 2005 Feb 8; 64(3):542-4.

Harno $\mathrm{H}$, Hirvonen $\mathrm{T}$, Kaunisto MA, Aalto $\mathrm{H}$, Levo $\mathrm{H}$, Isotalo $\mathrm{E}$, Kallela M, Kaprio J, Palotie A, Wessman M, Färkkilä M. Subclinical vestibulocerebellar dysfunction in migraine with and without aura. Neurology. 2003 Dec 23; 61(12):1748-52.

Headache Classification Subcommittee of the International Headache Society. The international classification of headache disorders, 2nd edition. Cephalalgia. 2004 24(Suppl 1):1-160.

Herrmann A, Braathen GJ, Russell MB. Episodic ataxias. Tidsskr Nor Laegeforen. 2005 Aug 11;125(15):2005-7.

Hirokawa S. Normal gait characteristics under temporal and distance constraints. J Biomed Eng 1989; 11: 449-456.

Jen J. Familial Episodic Ataxias and Related Ion Channel Disorders. Curr Treat Options Neurol. 2000 Sep; 2(5):429-431. 
Jen JC, Graves TD, Hess EJ, Hanna MG, Griggs RC, Baloh RW; CINCH investigators. Primary episodic ataxias: diagnosis, pathogenesis and treatment. Brain. 2007 Oct; 130(Pt 10):2484-93. Epub 2007 Jun 15.

Jen JC, Kim GW, Dudding KA, Baloh RW. No mutations in CACNA1A and ATP1A2 in probands with common types of migraine. Arch Neurol. 2004 Jun;61(6):926-8.

Kirchmann M, Thomsen LL, Olesen J. The CACNA1A and ATP1A2 genes are not involved in dominantly inherited migraine with aura. Am J Med Genet B Neuropsychiatr Genet. 2006 Apr 5;141B(3):250-6.

Kullmann DM. The neuronal channelopathies. Brain. 2002 Jun; 125(Pt 6):1177-95.

Kors EE, Haan J, Frants RR, Ferrari MD. From gene to disease; from CACNA1A to migraine. Ned Tijdschr Geneeskd. 2001 Feb 10;145(6):266-7.

Kors EE, Haan J, Giffin NJ, Pazdera L, Schnittger C, Lennox GG, Terwindt GM, Vermeulen FL, Van den Maagdenberg AM, Frants RR, Ferrari MD. Expanding the phenotypic spectrum of the CACNA1A gene T666M mutation: a description of 5 families with familial hemiplegic migraine. Arch Neurol. 2003 May; 60(5):684-8.

Lea RA, Shepherd AG, Curtain RP, Nyholt DR, Quinlan S, Brimage PJ, Griffiths LR. A typical migraine susceptibility region localizes to chromosome $1 \mathrm{q} 31$. Neurogenetics. 2002 Mar;4(1):17-22.

Nudler S, Piriz J, Urbano FJ, Rosato-Siri MD, Renteria ES, Uchitel OD. Ca2+ channels and synaptic transmission at the adult, neonatal, and P/Q-type deficient neuromuscular junction. Ann N Y Acad Sci. 2003 Sep; 998:11-7. 
Manto MU. The wide spectrum of spinocerebellar ataxias (SCAs). Cerebellum. $2005 ; 4(1): 2-6$.

Marti S, Baloh RW, Jen JC, Straumann D, Jung HH Progressive cerebellar ataxia with variable episodic symptoms--phenotypic diversity of R1668W CACNA1A mutation. Eur Neurol. 2008;60(1):16-20. Epub 2008 Apr 25.

Mintz IM, Adams ME, Bean BP. P-type calcium channels in rat central and peripheral neurons. Neuron. 1992 Jul; 9(1):85-95.

Montagna $\mathrm{P}$ The physiopathology of migraine: the contribution of genetics. Neurol Sci. 2004 Oct; 25 Suppl 3:S93-6.

Mori Y, Friedrich T, Kim MS, Mikami A, Nakai J, Ruth P, Bosse E, Hofmann F, Flockerzi V, Furuichi $\mathrm{T}$, et al. Primary structure and functional expression from complementary DNA of a brain calcium channel. Nature. 1991 Apr 4;350(6317):398402.

Ophoff RA, Terwindt GM, Vergouwe MN, Frants RR, Ferrari MD. Familial hemiplegic migraine: involvement of a calcium neuronal channel. Neurologia. 1997 Dec;12 Suppl $5: 31-7$

Østergaard E, Thomsen LL, Russell MB. Molecular genetic findings in migraine. Ugeskr Laeger. 2001 Nov 5;163(45):6260-5.

Pietrobon D. Calcium channels and channelopathies of the central nervous system. Mol Neurobiol. 2002 Feb;25(1):31-50. 
Pietrobon D. Function and dysfunction of synaptic calcium channels: insights from mouse models. Curr Opin Neurobiol. 2005 Jun; 15(3):257-65.

Pietrobon D. Migraine: new molecular mechanisms. Neuroscientist. 2005 Aug;11(4):373-86.

Rajakulendran S, Schorge S, Kullmann DM, Hanna MG. Episodic ataxia type 1: a neuronal potassium channelopathy. Neurotherapeutics. 2007 Apr; 4(2):258-66.

Russell MB, Iselius L, Olesen J. Migraine without aura and migraine with aura are inherited disorders. Cephalalgia. 1996 Aug;16(5):305-9.

Russell MB, Olesen J. The genetics of migraine without aura and migraine with aura. Cephalalgia. 1993 Aug;13(4):245-8.

Sanchez-Del-Rio M, Reuter U, Moskowitz MA. New insights into migraine pathophysiology. Curr Opin Neurol. 2006 Jun;19(3):294-8.

Sándor PS, Afra J, Proietti Cecchini AP, Albert A, Schoenen J. From neurophysiology to genetics: cortical information processing in migraine underlies familial influences--a novel approach. Funct Neurol. 2000; 15 Suppl 3:68-72.

Sándor PS, Afra J, Proietti-Cecchini A, Albert A, Schoenen J. Familial influences on cortical evoked potentials in migraine. Neuroreport. 1999 Apr 26;10(6):1235-8.

Sándor PS, Ambrosini A, Agosti RM, Schoenen J. Genetics of migraine: possible links to neurophysiological abnormalities. Headache. 2002 May; 42(5):365-77.

Sándor PS, Mascia A, Seidel L, de Pasqua V, Schoenen J. Subclinical cerebellar impairment in the common types of migraine: a three-dimensional analysis of reaching movements. Ann Neurol. 2001 May; 49(5):668-72. 
Schmitz N, Admiraal-Behloul F, Arkink EB, Kruit MC, Schoonman GG, Ferrari MD, van Buchem MA. Attack frequency and disease duration as indicators for brain damage in migraine. Headache. 2008 Jul; 48(7):1044-55. Epub 2008 May 9.

Schoenen J, Ambrosini A, Sándor PS, Maertens de Noordhout A. Evoked potentials and transcranial magnetic stimulation in migraine: published data and viewpoint on their pathophysiologic significance. Clin Neurophysiol. 2003 Jun; 114(6):955-72.

Seet RC, Lim EC. A case of intermittent ataxia associated with migraine headaches. CMAJ. 2007 Sep 11;177(6):565-7.

Stam AH, van den Maagdenberg AM, Haan J, Terwindt GM, Ferrari MD. Genetics of migraine: an update with special attention to genetic comorbidity. Curr Opin Neurol. 2008 Jun; 21(3):288-93.

Stolze H. Der Gang im Alter und bei Morbus Parkinson. 1995; S. 50-55

Takahashi T, Igarashi S, Tsuji S. Familial hemiplegic migraine Clin Calcium. 2001 Nov; 11(11):1460-3.

Terakawa H, Abe K, Watanabe Y, Nakamura M, Fujita N, Hirabuki N, Yanagihara T. Proton magnetic resonance spectroscopy (1H MRS) in patients with sporadic cerebellar degeneration. J Neuroimaging. 1999 Apr;9(2):72-7.

Terwindt GM, Ophoff RA, van Eijk R, Vergouwe MN, Haan J, Frants RR, Sandkuijl LA, Ferrari MD; Dutch Migraine Genetics Research Group. Involvement of the CACNA1A gene containing region on $19 p 13$ in migraine with and without aura. Neurology. 2001 Apr 24;56(8):1028-32. 
Terwindt GM, Ophoff RA, Haan J, Sandkuijl LA, Frants RR, Ferrari MD. Migraine, ataxia and epilepsy: a challenging spectrum of genetically determined calcium channelopathies. Dutch Migraine Genetics Research Group. Eur J Hum Genet. 1998 Jul-Aug; 6(4):297-307.

Thomsen LL, Olesen J, Russell MB. Familial hemiplegic migraine. Ugeskr Laeger. 1998 Sep 7;160(37):5325-9.

Tonelli A, D'Angelo MG, Salati R, Villa L, Germinasi C, Frattini T, Meola G, Turconi AC, Bresolin N, Bassi MT. Early onset, non fluctuating spinocerebellar ataxia and a novel missense mutation in CACNA1A gene. J Neurol Sci. 2006 Feb 15; 241(1-2):137. Epub 2005 Dec 2.

Trouillas $\mathrm{P}$, et al. International Cooperative Ataxia Rating Scale for pharmacological assessment of the cerebellar syndrome. The Ataxia Neuropharmacology Committee of the World Federation of Neurology. J Neurol Sci. 1997 Feb 12; 145(2):205-11.

Urbano FJ, Pagani MR, Uchitel OD. Calcium channels, neuromuscular synaptic transmission and neurological diseases. J Neuroimmunol. 2008 Sep 15; 201202C:136-144. Epub 2008 Aug 3.

Vincent M, Hadjikhani N. The cerebellum and migraine. Headache. 2007 Jun;47(6):820-33. 


\section{Danksagung}

Herrn Prof. Dr. med. G. Deuschl, Direktor der Klinik für Neurologie am Universitätsklinikum Schleswig Holstein Campus Kiel danke ich für die Möglichkeit der uneingeschränkten Durchführung der nötigen Untersuchungen, Messungen und Analysen in seiner Klinik.

Mein besonderer Dank gilt Herrn Prof. Dr. med. H. Stolze für die gute Betreuung während der Arbeit sowie für die Auswahl und Überlassung des Themas zu dieser Doktorarbeit.

Des Weiteren danke ich allen Patienten der Migräne-Sprechstunde am Universitätsklinikum Schleswig-Holstein Campus Kiel sowie allen Freunden und Freiwilligen für Ihre Bereitschaft, an der Studie teilzunehmen.

Allen Mitarbeitern des Motoriklabors der Klinik für Neurologie am UK-SH für Ihre Unterstützung und Bereitschaft, insbesondere Herrn Birger Streckel für die Hilfe bei der Auswertung der Gangparameter mittels des von inm programmierten AnalyseProgramms GaitLab Version 2.1.

Weiterhin danke ich der Firma "Qualisys-Motion Capture Systems" für die unkomplizierte Zusammenarbeit und stetige Bemühungen zur Optimierung der Messdaten.

Zuletzt danke ich meinem guten und langjährigen Freund Eike Christian Rehwald für seine Unterstützung bei der graphischen Umsetzung und Darstellung der Ergebnisse. 


\section{Lebenslauf}

Am 24. Juni 1981 wurde ich als Sohn des Lehrers Hermann Timm sowie der Bürokauffrau Rita Timm in Aurich geboren. Nach Vollendung der Grundschule wechselte ich an die Integrierte Gesamtschule Aurich-West und bestand im Juni 2000 die Allgemeine Hochschulreife.

Von Juli 2000 bis zum Mai 2001 leistete ich einen 11-monatigen Zivildienst beim Rettungsdienst des Landkreises Aurich und bestand in dieser Zeit meine Ausbildung zum Rettungssanitäter an der Lifetime $\mathrm{GmbH}-$ Rettungsschule in Norden.

Im Oktober 2001 begann ich das Studium der Humanmedizin an der Medizinischen Fakultät der Christian-Albrechts-Universität zu Kiel. Nach bestandenem Physikum 2003 folgte nach erfolgreicher Absolvierung des klinischen Hauptstudiums das Praktische Jahr mit dem Wahlfach Neurologie, welches an der Klinik für Neurologie am Universitätsklinikum Schleswig-Holstein geleistet wurde. Im Juni 2008 erhielt ich nach bestandener ärztlicher Prüfung die Approbation als Arzt.

Seit November 2008 arbeite ich in der Anstellung als Assistenzarzt in der Abteilung für Neurologie der Asklepios Klinik Altona unter ärztlicher Leitung von Herrn Prof. Dr. med. Röther. 